\title{
Local Palais-Smale sequences for the Willmore functional
}

\author{
YAnn Bernard And Tristan Rivière
}

\begin{abstract}
Using the reformulation in divergence form of the Euler-Lagrange equation for the Willmore functional as it was developed in the second author's paper [24], we study the limit of a local PalaisSmale sequence of weak Willmore immersions with locally squareintegrable second fundamental form. We show that the limit immersion is smooth and that it satisfies the conformal Willmore equation: it is a critical point of the Willmore functional restricted to infinitesimal conformal variations.
\end{abstract}

\section{Introduction}

Let $\Sigma$ be a closed oriented surface without boundary immersed in $\mathbb{R}^{m \geq 3}$ through the action of a smooth positive immersion $\vec{\Phi}$. Its Willmore energy is the functional

$$
W(\vec{\Phi}(\Sigma)):=\int_{\Sigma}|\vec{H}|^{2} d \mu_{g}
$$

where $\vec{H} \in \mathbb{R}^{m}$ is the mean curvature vector, and $d \mu_{g}$ is the area form of the metric $g$ induced on $\vec{\Phi}(\Sigma)$ via the canonical metric on $\mathbb{R}^{m}$.

The critical points of (1.1) for perturbations of the form $\vec{\Phi}+t \vec{\xi}$, where $\vec{\xi}$ is an arbitrary compactly supported smooth map on $\Sigma$ into $\mathbb{R}^{m}$ are known as Willmore surfaces. Examples of Willmore surfaces include, among many others, minimal surfaces (which are absolute minimizers), and round spheres. Further examples may be found in [20,36], and the references therein.

The Willmore functional naturally arises in various areas of science $[11$, $13,15,16,21]$. Its importance is largely due to its invariance under conformal transformations $[9,34]$. As the second author of the present paper showed in [23], the Euler-Lagrange equation arising from a two-dimensional conformally invariant Lagrangian with quadratic growth can be written in divergence form. These "conservation laws" fostered within variational problems involving conformally invariant Lagrangians offer a significant analytical help. The general ideas introduced in [23] are led to fruition in [24], where 
conservation laws relative to the Willmore functional are developed and successfully applied to produce a variety of interesting results. Our present work stems from this alternative formulation of the Willmore equation.

Owing to the Gauß-Bonnet theorem, we note that the Willmore energy (1.1) may be equivalently expressed as

$$
W(\vec{\Phi}(\Sigma))=\int_{\Sigma}|\overrightarrow{\mathbb{I}}|_{g}^{2} d \mu_{g}+\pi \chi(\Sigma)
$$

where $\overrightarrow{\mathbb{I}}$ is the second fundamental form, and $\chi(\Sigma)$ is the Euler characteristic of $\Sigma$. Since the latter is a topological invariant, from the variational point of view, Willmore surfaces are the critical points of the energy $\int_{\Sigma}|\overrightarrow{\mathbb{I}}|_{g}^{2} d \mu_{g}$. It thus appears natural to restrict our attention on a weak ${ }^{1}$ immersion $\vec{\Phi}: \Sigma \rightarrow \mathbb{R}^{m}$ whose second fundamental forms are locally square-integrable. Such immersions were characterized in [30, 31], and in [19]. Frédéric Hélein (see Theorem 5.1.1 in [14]) showed that, locally about every point on $\Sigma$, there exist an open disk $D$ and a homeomorphism $\zeta$ of $D$ such that $\vec{\Phi} \circ \zeta$ is a conformal bilipschitz immersion. In this parametrization, the induced metric $g$ on $D^{2}$ is continuous; and the Gauß map $\vec{n}$ lies in $W^{1,2}\left(D^{2}, G r_{m-2}\left(\mathbb{R}^{m}\right)\right)$, relative to the induced metric $g$. Slightly abusing notation, we continue to denote $\vec{\Phi} \circ \zeta$ by $\vec{\Phi}$. Owing to the invariance of the Willmore energy under reparametrization, we shall henceforth assume that $\vec{\Phi}$ is a conformal weak immersion of the unit disc $D^{2}=\left\{x=\left(x_{1}, x_{2}\right) \in \mathbb{R}^{2} ; x_{1}^{2}+x_{2}^{2}<1\right\}$ :

$$
\left|\partial_{x_{1}} \vec{\Phi}\right|=\mathrm{e}^{\lambda}=\left|\partial_{x_{2}} \vec{\Phi}\right| \quad \text { and } \quad \partial_{x_{1}} \vec{\Phi} \cdot \partial_{x_{2}} \vec{\Phi}=0
$$

where $\lambda$ is the conformal parameter. An elementary computation shows that

$$
d \mu_{g}=\mathrm{e}^{2 \lambda} d x \quad \text { and } \quad|\nabla \vec{n}|^{2} d x=\mathrm{e}^{2 \lambda}|\overrightarrow{\mathbb{I}}|_{g}^{2} d x=|\overrightarrow{\mathbb{I}}|_{g}^{2} d \mu_{g}
$$

Hence, by hypothesis, we see that $\vec{n} \in W^{1,2}\left(D^{2}\right)$. Rescaling if necessary, we shall always assume that

$$
\int_{D^{2}}|\nabla \vec{n}|^{2} d x \leq \varepsilon
$$

where the adjustable parameter $\varepsilon$ is chosen to fit our various needs (we will need it to be "small enough" in Theorem 2.2).

\footnotetext{
${ }^{1}$ cf. [24] for details.
} 
We set

$$
\vec{e}_{j}:=\mathrm{e}^{-\lambda} \partial_{x_{j}} \vec{\Phi} \quad \text { for } \quad j \in\{1,2\} .
$$

As $\vec{\Phi}$ is conformal, $\left\{\vec{e}_{1}(x), \vec{e}_{2}(x)\right\}$ forms an orthonormal basis of the tangent space $T_{\vec{\Phi}(x)} \vec{\Phi}\left(D^{2}\right)$. Owing to the topology of $D^{2}$, there exists for almost every $x \in D^{2}$ a positively oriented orthonormal basis $\left\{\vec{n}_{1}, \ldots, \vec{n}_{m-2}\right\}$ of the normal space $N_{\vec{\Phi}(x)} \vec{\Phi}\left(D^{2}\right)$, such that $\left\{\vec{e}_{1}, \vec{e}_{2}, \vec{n}_{1}, \ldots, \vec{n}_{m-2}\right\}$ forms a basis of $T_{\vec{\Phi}(x)} \mathbb{R}^{m}$. From the Plücker embedding, realizing the Grassmannian $\operatorname{Gr}_{m-2}\left(\mathbb{R}^{m}\right)$ as a submanifold of the projective space of the $(m-2)$ th exterior power $\mathbb{P}\left(\bigwedge^{m-2} \mathbb{R}^{m}\right)$, we can represent the Gauß map as the $(m-2)$-vector $\vec{n}=$ $\bigwedge_{\alpha=1}^{m-2} \vec{n}_{\alpha}$. Via the Hodge operator $\star$, we identify vectors and $(m-1)$-vectors in $\mathbb{R}^{m}$, namely:

$$
\star\left(\vec{n} \wedge \vec{e}_{1}\right)=\vec{e}_{2}, \quad \star\left(\vec{n} \wedge \vec{e}_{2}\right)=-\vec{e}_{1}, \quad \star\left(\vec{e}_{1} \wedge \vec{e}_{2}\right)=\vec{n} .
$$

In this notation, the second fundamental form is expressed as

$$
\overrightarrow{\mathbb{I}}=\sum_{\alpha, i, j} h_{i j}^{\alpha} \vec{n}_{\alpha}\left(\vec{e}_{i}\right)^{*} \otimes\left(\vec{e}_{j}\right)^{*}, \quad \text { where } \quad h_{i j}^{\alpha}:=-\mathrm{e}^{-\lambda} \vec{e}_{i} \cdot \partial_{x_{j}} \vec{n}_{\alpha}
$$

The mean curvature vector is

$$
\vec{H}=\sum_{\alpha=1}^{m-2} H^{\alpha} \vec{n}_{\alpha}=\frac{1}{2} \sum_{\alpha=1}^{m-2}\left(h_{11}^{\alpha}+h_{22}^{\alpha}\right) \vec{n}_{\alpha} .
$$

The Willmore equation satisfied by Willmore surfaces [32] becomes

$$
\Delta_{\perp} \vec{H}+\sum_{\alpha, \beta, i, j} h_{i j}^{\alpha} h_{i j}^{\beta} H^{\beta} \vec{n}_{\alpha}-2|\vec{H}|^{2} \vec{H}=0
$$

with

$$
\Delta_{\perp} \vec{H}:=\mathrm{e}^{-2 \lambda} \pi_{\vec{n}} \operatorname{div}\left(\pi_{\vec{n}}(\nabla \vec{H})\right),
$$

and $\pi_{\vec{n}}$ is the projection onto the normal space spanned by $\left\{\vec{n}_{\alpha}\right\}_{\alpha=1}^{m-2}$.

The Willmore equation (1.2) is a fourth-order nonlinear equation (in the coefficients of the induced metric, which depends on $\vec{\Phi}$ ). With respect to the coefficients $H^{\alpha}$ of the mean curvature vector, it is actually a strongly coupled system whose study is particularly challenging. In codimension 1, there is one equation for the scalar curvature; in higher codimension, the situation becomes significantly more complicated, and one must seek different techniques to approach (1.2). Fortunately, in a conformal parametrization, 
the second authors showed that it is possible ${ }^{2}$ to recast the system (1.2) in an equivalent, but analytically more suitable form [24]. Namely, there holds

$$
\operatorname{div}\left(\nabla \vec{H}-3 \pi_{\vec{n}}(\nabla \vec{H})+\star\left(\nabla^{\perp} \vec{n} \wedge \vec{H}\right)\right)=0
$$

where $\nabla^{\perp}=\left(-\partial_{x_{2}}, \partial_{x_{1}}\right)$. This reformulation in divergence form of the Willmore equation is the starting point of our analysis of local Palais-Smale sequences.

\section{Main results}

\subsection{Local Palais-Smale sequences}

Tom Willmore conjectured [35] that the Clifford torus minimizes, up to Möbius transformations, the Willmore energy in the class of smooth immersed tori in $\mathbb{R}^{3}$. A satisfactory demonstration of this assertion has yet to be found. Amid the works aimed at solving this problem, one fundamental property of the Willmore functional was brought into light by Leon Simon [26]: for each $m \geq 3$, there exists a compact embedded real analytic torus in $\mathbb{R}^{m}$ which minimizes the Willmore energy in the class of compact, genus 1, embedded surfaces without boundary. Unfortunately, it remains unknown whether this minimizer is the Willmore torus. Simon's proof is variational and devises a minimizing sequence for the Willmore functional. This is one instance in which studying and understanding minimizing sequences of the Willmore functional are essential. Yet more generally, it is interesting to investigate Palais-Smale sequences.

We open our considerations with an "empirical" observation which will hopefully convince the reader that the results derived in [24] offer a suitable framework to acquire information on Palais-Smale sequences of Willmore surfaces. Let us consider a conformal weak Willmore immersion $\vec{\Phi}$ from the flat disk $D^{2}$ into $\mathbb{R}^{m}$ with square-integrable second fundamental form. The "divergence-free" form given in (1.3) implies that, up to an additive constant, it is possible to define a map $\vec{L}$ satisfying

$$
\nabla^{\perp} \vec{L}:=\nabla \vec{H}-3 \pi_{\vec{n}}(\nabla \vec{H})+\star\left(\nabla^{\perp} \vec{n} \wedge \vec{H}\right)
$$

\footnotetext{
${ }^{2}$ this procedure requires to choose the normal frame $\left\{\vec{n}_{\alpha}\right\}$ astutely. See [24] for details.
} 
In [24], it is shown that the following system holds: ${ }^{3}$

$$
\left\{\begin{array}{l}
\nabla \vec{\Phi} \cdot \nabla^{\perp} \vec{L}=0, \\
\nabla \vec{\Phi} \wedge \nabla^{\perp} \vec{L}=2(-1)^{m} \nabla\left(\star ( \vec { n } \llcorner \vec { H } ) ) \left\llcorner\nabla^{\perp} \vec{\Phi} .\right.\right.
\end{array}\right.
$$

The Hodge operator $\star$ and the contraction operator $L$ commute with all differential operators. Accordingly, the terms appearing in the system (2.2) enjoy a peculiar property: more than mere "products of derivatives," they can be written in divergence form. This structural feature has the analytical advantage of being robust under weak limiting process. Hence it is legitimate to hope that a local Palais-Smale sequence of conformal weak Willmore immersions with, say, uniformly bounded square-integrable second fundamental forms, converges to an element $\vec{\Phi}$ satisfying the system (2.2) for some function $\vec{L}$ (which may or may not be related to $\vec{\Phi}$ via (2.1)).

This is essentially the result that we shall establish. Prior to stating it precisely, it is necessary to specifically define the notion of local Palais-Smale sequence for the Willmore functional.

Definition 2.1. Let $\left(\vec{\Phi}_{k}\right)$ be a sequence of conformal immersions from the unit disc $D^{2}$ into $\mathbb{R}^{m}$ such that

$$
\left\|\vec{\Phi}_{k}\right\|_{W^{2,2} \cap W^{1, \infty}}+|\log | \nabla \vec{\Phi}_{k}|| \leq C
$$

holds uniformly in $k$ for some positive constant $C$. Denoting, respectively, by $\vec{n}_{k}$ and $\vec{H}_{k}$ the Gauß map and the mean curvature vector associated with the immersion $\vec{\Phi}_{k}$, we set

$$
Q_{k}:=\nabla \vec{H}_{k}-3 \pi_{\vec{n}_{k}}\left(\nabla \vec{H}_{k}\right)+\star\left(\nabla^{\perp} \vec{n}_{k} \wedge \vec{H}_{k}\right)
$$

The sequence $\left(\vec{\Phi}_{k}\right)$ is locally Palais-Smale if, in addition to (2.3), it satisfies

$$
\operatorname{div} Q_{k} \longrightarrow 0 \quad \text { strongly in } \quad\left(W^{2,2} \cap W^{1, \infty}\right)^{\prime}\left(D^{2}\right) .
$$

Remark 2.1. The first summand in (2.3) guarantees that the sequence $\left(\vec{\Phi}_{k}\right)$ is uniformly bounded in the appropriate space. Recall that the conformal parameter satisfies

$$
\lambda_{k}=\frac{1}{2} \log \left(\frac{1}{2}\left|\nabla \vec{\Phi}_{k}\right|^{2}\right) .
$$

\footnotetext{
${ }^{3}$ refer to the Introduction or Appendix A for the notation.
} 
Accordingly, the second summand in (2.3) insures that $\left(\lambda_{k}\right)$ remains uniformly bounded, so that the sequence of immersions does not degenerate and collapse. This condition is added here only for technical simplicity. As explained in [25], it is satisfied away from finitely many points, as one considers sequences of conformal immersions with uniformly bounded Willmore energy modulo some composition with elements from the Möbius group in $\mathbb{R}^{m}$.

The following result provides a first description of the limit of a PalaisSmale sequence of the Willmore functional.

Theorem 2.1. Let $\left(\vec{\Phi}_{k}\right)$ be a local Palais-Smale sequence of conformal immersions from the unit disc $D^{2}$ into $\mathbb{R}^{m}$. There exist a conformal weak immersion $\vec{\Phi} \in\left(W^{2,2} \cap W^{1, \infty}\right)\left(D^{2}\right)$ and an element ${ }^{4} \vec{L} \in L^{2, \infty}\left(D^{2}\right)$ such that, up to extraction of a subsequence,

$$
\vec{\Phi}_{k} \longrightarrow \vec{\Phi} \quad \text { in } \quad W^{2,2}\left(D^{2}\right)
$$

and the system

$$
\left\{\begin{array}{l}
\nabla \vec{\Phi} \cdot \nabla^{\perp} \vec{L}=0, \\
\nabla \vec{\Phi} \wedge \nabla^{\perp} \vec{L}=-2 \nabla \vec{\Phi} \wedge \nabla \vec{H}
\end{array}\right.
$$

holds in the sense of distributions, where $\vec{H}$ denotes the mean curvature vector associated with $\vec{\Phi}$.

Remark 2.2. This result is one of the fundamental tools used in [25] to construct minimizers (and critical points) of the Willmore functional under various prescribed constraints (fixed conformal class, fixed surface area and fixed volume, etc.) and to apply the principles of the calculus of variations (e.g. minimizing principles, Ekeland variational principle, mountain-pass lemma, etc.).

The apparent difference between the systems (2.2) and (2.5) is fictitious only. Indeed, one verifies ${ }^{5}$ the identity

$$
\nabla \vec{\Phi} \wedge \nabla \vec{H}=(-1)^{m-1} \nabla\left(\star ( \vec { n } \llcorner \vec { H } ) ) \left\llcorner\nabla^{\perp} \vec{\Phi} .\right.\right.
$$

\footnotetext{
${ }^{4}$ The space $L^{2, \infty}$ is defined in Appendix A.2.

${ }^{5}$ cf. Equation (2.49) in the paper [24].
} 


\subsection{The conformal Willmore equation}

The convergence result stated in Theorem 2.1 begs the following question: if an immersion $\vec{\Phi}$ satisfies, for some function $\vec{L}$, the system (2.5), is it true that $\vec{\Phi}$ is Willmore? Unfortunately, and perhaps surprisingly, the answer is negative (or rather, "not quite"). Nevertheless, the following description can be obtained.

Theorem 2.2. Let $\vec{\Phi} \in W^{2,2} \cap W^{1, \infty}$ be a conformal weak immersion from the unit disc $D^{2}$ into $\mathbb{R}^{m}$, and such that

$$
\int_{D^{2}}|\nabla \vec{n}|^{2} \leq \varepsilon
$$

for some $\varepsilon>0$ small enough. Then there exists $\vec{L} \in L^{2, \infty}\left(D^{2}\right)$ satisfying (2.5) if and only if $\vec{\Phi}$ is smooth and there holds

$$
\mathrm{e}^{2 \lambda}\left[\Delta_{\perp} \vec{H}+\sum_{\alpha, \beta, i, j} h_{i j}^{\alpha} h_{i j}^{\beta} H^{\beta} \vec{n}_{\alpha}-2|\vec{H}|^{2} \vec{H}\right]=\Im\left(\vec{H}_{0} f\right),
$$

for some holomorphic function $f$. The Weingarten operator is defined via

$$
\vec{H}_{0}:=\frac{1}{2}\left(h_{11}^{\alpha}-h_{22}^{\alpha}+2 i h_{12}^{\alpha}\right) \vec{n}_{\alpha}
$$

Although the amount of information on the holomorphic function $f$ is rather limited, we note that the way it appears on the right-hand side of (2.7) makes it play the rôle of a Lagrange multiplier in the Willmore equation (1.2). This observation enables one to give a natural geometric interpretation of $f$ in the context of Teichmüller theory (cf. [6]).

The Willmore functional being conformally invariant, the left-hand side of (2.7) remains unchanged under a holomorphic change of coordinates. An elementary computation reveals that $\frac{1}{2} \vec{H}_{0}$ is the coefficient ${ }^{6}$ of $d z \otimes d z$ in the second fundamental form. Looking at the right-hand side of (2.7), we deduce that $f$ must be the coordinate of a section $f(z) \partial_{z} \otimes \partial_{z}$. There is thus a one-to-one correspondence between the vector space to which $f$ belongs and the vector space $\mathcal{H}^{0}(\Sigma)$ of holomorphic quadratic differentials. Let $\mathfrak{g}$ be

\footnotetext{
${ }^{6}$ the variable $z$ is defined as $x_{1}+\mathrm{i} x_{2}$, where $\left(x_{1}, x_{2}\right)$ are the usual coordinates on the unit disc for the flat metric.
} 
the genus of the immersed surface $\Sigma$. From the Riemann-Roch theorem (cf. Corollary 5.4.2 in [18]), the space $\mathcal{H}^{0}(\Sigma)$ satisfies

$$
\operatorname{dim}_{\mathbb{C}} \mathcal{H}^{0}(\Sigma)= \begin{cases}0, & \mathfrak{g}=0 \\ 1, & \mathfrak{g}=1 \\ 3(\mathfrak{g}-1), & \mathfrak{g} \geq 2\end{cases}
$$

Equation (2.7) is not novelty; it has been studied in various contexts. Immersions that satisfy (2.7) are sometimes known as constrained Willmore immersions, such as in [6], where it is shown that (2.7) is the EulerLagrange equation deriving from the Willmore functional (1.1) under smooth compactly supported infinitesimal conformal variations. The corresponding critical points are the conformal-constrained Willmore surfaces. This notion clearly generalizes that of a Willmore surface, obtained via all smooth compactly supported infinitesimal variations. Conformal-constrained Willmore surfaces form a Möbius invariant class of surfaces fostering remarkable properties, some of which are studied in $[6,8,22]$. In the latter, it is in particular established that every constant mean curvature surface in a three-dimensional space form is conformal-constrained Willmore. We have chosen to refer to (2.7) as the conformal Willmore equation, rather than as the somewhat vaguer term "constrained Willmore equation." There are indeed many ways to constrain the variations of the Willmore functional (e.g. restrictions on the volume and surface area in the Helfrich model). The adjective "conformal" appears more descriptive in our situation.

\section{Proofs of the theorems}

\subsection{Proof of Theorem 2.1}

Let us set

$$
Q_{k}:=\nabla \vec{H}_{k}-3 \pi_{\vec{n}}\left(\nabla \vec{H}_{k}\right)+\star\left(\nabla^{\perp} \vec{n}_{k} \wedge \vec{H}_{k}\right) .
$$

We suppose that

$$
\operatorname{div} Q_{k} \longrightarrow 0 \quad \text { strongly in } \quad\left(W^{2,2} \cap W^{1, \infty}\right)^{\prime}\left(D^{2}\right)
$$

and

$$
\left\|\vec{\Phi}_{k}\right\|_{W^{2,2} \cap W^{1, \infty}} \leq C \quad \text { uniformly, for some constant } C>0 .
$$


By definition and from (3.3), it is not hard to see that the sequences $\left(\vec{n}_{k}\right)$, $\left(\vec{H}_{k}\right)$ and $\left(Q_{k}\right)$ are uniformly bounded in $W^{1,2}, L^{2}$ and $\mathbb{R}^{2} \otimes\left(W^{-1,2}+L^{1}\right)$, respectively. These facts shall be recurrently used in the sequel.

We begin our study with an elementary result.

Lemma 3.1. There holds

$$
\left\{\begin{array}{l}
\vec{\Phi}_{k} \cdot \operatorname{div} Q_{k} \longrightarrow 0 \\
\vec{\Phi}_{k} \wedge \operatorname{div} Q_{k} \longrightarrow 0
\end{array} \quad \text { in } \quad \mathcal{D}^{\prime}\left(D^{2}\right)\right.
$$

Proof. Both convergences are obtained in analogous fashions (interchanging the dot and wedge products), so we concentrate only on the first one. For notational convenience, we set

$$
X:=W^{2,2} \cap W^{1, \infty},
$$

and $X_{0}$ is the space of elements of $X$ with vanishing trace on $\partial D^{2}$. Let $u$ be an arbitrary element of $\mathcal{D}=C_{c}^{\infty}\left(D^{2}\right)$. The Sobolev embedding theorem guarantees that the elements $\vec{\Phi}_{k}$ of $X$ are Hölder continuous on $\overline{D^{2}}$. Since in addition $X$ is an intersection of Sobolev spaces, it is clear that $\vec{\Phi}_{k} u$ is an element of $X_{0}$. More precisely, from (3.3),

$$
\left\|\vec{\Phi}_{k} u\right\|_{X_{0}} \lesssim\left\|\vec{\Phi}_{k}\right\|_{X}\|u\|_{\mathcal{D}} \leq C\|u\|_{\mathcal{D}}
$$

We can make sense of $\vec{\Phi}_{k} \cdot T_{k}$ as a distribution via

$$
\left\langle\vec{\Phi}_{k} \cdot \operatorname{div} Q_{k}, u\right\rangle_{\mathcal{D}^{\prime}, \mathcal{D}}:=\left\langle\operatorname{div} Q_{k}, \vec{\Phi}_{k} u\right\rangle_{X_{0}^{\prime}, X_{0}}
$$

It then follows immediately from (3.2) and (3.4) that $\vec{\Phi}_{k} \cdot \operatorname{div} Q_{k}$ converges to zero in the sense of distributions.

By hypothesis, $\operatorname{div} Q_{k}$ is a bounded linear functional on $X$ (with $X$ as in the proof of the previous lemma). According to (A.3), the space $W_{0}^{2,(2,1)}$ is continuously embedded into $X$. Hence $\operatorname{div} Q_{k}$ belongs to $W^{-2,(2, \infty)}$, dual of $W_{0}^{2,(2,1)}$. Proposition A.1 grants therefore the existence of an element $P_{k}$ in $\mathbb{R}^{2} \otimes W^{-1,(2, \infty)}$ satisfying

$$
\operatorname{div} Q_{k}=\operatorname{div} P_{k}
$$

and

$$
\left\|P_{k}\right\|_{W^{-1,(2, \infty)}} \leq\left\|\operatorname{div} Q_{k}\right\|_{W^{-2,(2, \infty)}}+\delta_{k},
$$

for some positive constant $\delta_{k}$ arbitrarily chosen. 
We next establish a useful result.

Lemma 3.2. There holds

$$
\left\{\begin{array}{l}
\vec{\Phi}_{k} \cdot P_{k} \longrightarrow 0 \\
\vec{\Phi}_{k} \wedge P_{k} \longrightarrow 0
\end{array} \quad \text { in } \quad \mathcal{D}^{\prime}\left(D^{2}\right) .\right.
$$

Proof. Owing to (3.2) and (3.6), the sequence $\left(P_{k}\right)$ converges strongly to zero in $W^{-1,(2, \infty)} \equiv\left(W_{0}^{1,(2,1)}\right)^{\prime}$. Moreover, from (3.3) and (A.4), the sequence $\left(\vec{\Phi}_{k}\right)$ is uniformly bounded in $W^{1,(2,1)}$. The desired statements may now be reached by repeating mutatis mutandis the proof of Lemma 3.1, and letting $\delta_{k}$ tend to zero.

Identity (3.5) says that

$$
\operatorname{div}\left(Q_{k}-P_{k}\right)=0
$$

We have seen that $Q_{k}$ belongs to $\mathbb{R}^{2} \otimes\left(W^{-1,2}+L^{1}\right)$ and that $P_{k}$ belongs to $\mathbb{R}^{2} \otimes W^{-1,(2, \infty)}$. Hence, altogether, the difference $\left(Q_{k}-P_{k}\right)$ lies in $\mathbb{R}^{2} \otimes$ $\left(W^{-1,2}+W^{-1,(2, \infty)}+L^{1}\right)$. As proved in Lemma A.1, Equation (3.7) implies the existence of an element $\vec{L}_{k}$ in $L^{2, \infty}$ satisfying

$$
Q_{k}-P_{k}=\nabla^{\perp} \vec{L}_{k}
$$

Because all sequences involved are uniformly bounded, one notices that likewise the sequence $\left(\nabla^{\perp} \vec{L}_{k}\right)$ is uniformly bounded in $\mathbb{R}^{2} \otimes W^{-1,(2, \infty)}$.

Lemma 3.3. There holds

$$
\left\{\begin{array}{r}
\nabla \vec{\Phi}_{k} \cdot \nabla^{\perp} \vec{L}_{k} \longrightarrow 0 \\
\nabla \vec{\Phi}_{k} \wedge\left(\nabla^{\perp} \vec{L}_{k}+2 \nabla \vec{H}_{k}\right) \longrightarrow 0
\end{array} \quad \text { in } \quad \mathcal{D}^{\prime}\left(D^{2}\right) .\right.
$$

Proof. Observe that

$$
\nabla \vec{\Phi}_{k} \cdot P_{k}=\operatorname{div}\left(\vec{\Phi}_{k} \cdot P_{k}\right)-\vec{\Phi}_{k} \operatorname{div} P_{k}=\operatorname{div}\left(\vec{\Phi}_{k} \cdot P_{k}\right)-\vec{\Phi}_{k} \operatorname{div} Q_{k}
$$

Whence, the results of Lemmas 3.1 and 3.2 show that

$$
\nabla \vec{\Phi}_{k} \cdot P_{k} \longrightarrow 0 \quad \text { in } \quad \mathcal{D}^{\prime}\left(D^{2}\right)
$$

Similarly, one obtains

$$
\nabla \vec{\Phi}_{k} \wedge P_{k} \longrightarrow 0 \quad \text { in } \quad \mathcal{D}^{\prime}\left(D^{2}\right)
$$


From (A.13) in Appendix A, we have the identities

$$
\nabla \vec{\Phi}_{k} \cdot Q_{k}=0 \quad \text { and } \quad \nabla \vec{\Phi}_{k} \wedge Q_{k}=-2 \nabla \vec{\Phi}_{k} \wedge \nabla \vec{H}_{k}
$$

The desired statement now ensues by combining (3.8) to (3.10).

From the characterization provided in Proposition A.1, we can always arrange for the $L^{2, \infty}$-norm of $\vec{L}_{k}$ to come as close as we please to the $W^{-1,(2, \infty)}$-norm of $\nabla^{\perp} \vec{L}_{k}$. In particular, the sequence $\left(\vec{L}_{k}\right)$ is uniformly bounded in $L^{2, \infty}$. We may thus extract a weak* convergent subsequence (indexed for notational convenience as the original sequence) with

$$
\left\{\begin{array}{rll}
\vec{L}_{k} \stackrel{*}{\longrightarrow} \vec{L} & \text { in } & L^{2, \infty} \\
\nabla^{\perp} \vec{L}_{k} \stackrel{*}{\longrightarrow} g & \text { in } & \mathbb{R}^{2} \otimes W^{-1,(2, \infty)}
\end{array}\right.
$$

and $g=\nabla^{\perp} \vec{L}$ in the sense of distributions.

As the sequence $\left(\vec{\Phi}_{k}\right)$ is uniformly bounded in $W^{2,2} \cap W^{1, \infty}$ by hypothesis, the Banach-Alaoglu theorem yields a subsequence $\left(\vec{\Phi}_{k}\right)$ converging weak ${ }^{*}$ in $W^{2,2} \cap W^{1, \infty}$ to some element $\vec{\Phi}$. In turn, the compact embeddings (A.4) provided by the Rellich-Kondrachov theorem enable us to further extract a subsequence, still denoted $\left(\vec{\Phi}_{k}\right)$, satisfying the strong convergences

$$
\left\{\begin{array}{cll}
\vec{\Phi}_{k} \longrightarrow \vec{\Phi} & \text { in } & \bigcap_{p<\infty} W^{1, p}, \\
\nabla \vec{\Phi}_{k} \longrightarrow \nabla \vec{\Phi} & \text { in } \quad & \mathbb{R}^{2} \otimes \bigcap_{p<\infty} L^{p}
\end{array}\right.
$$

Convergences (3.11) and (3.12) imply the next result.

\section{Lemma 3.4.}

$$
\left\{\begin{array}{l}
\nabla \vec{\Phi}_{k} \cdot \nabla^{\perp} \vec{L}_{k} \longrightarrow \nabla \vec{\Phi} \cdot \nabla^{\perp} \vec{L} \\
\nabla \vec{\Phi}_{k} \wedge \nabla^{\perp} \vec{L}_{k} \longrightarrow \nabla \vec{\Phi} \wedge \nabla^{\perp} \vec{L}
\end{array} \quad \text { in } \quad \mathcal{D}^{\prime}\left(D^{2}\right) .\right.
$$

Proof. We shall only establish the first convergence, the second one being obtained mutatis mutandis. Owing to the general identity

$$
\operatorname{div}\left(a \nabla^{\perp} b\right)=\nabla a \cdot \nabla^{\perp} b
$$

it suffices to show that

$$
\vec{\Phi}_{k} \nabla^{\perp} \vec{L}_{k} \longrightarrow \vec{\Phi} \nabla^{\perp} \vec{L} \quad \text { in } \quad \mathcal{D}^{\prime}\left(D^{2}\right) .
$$


This is what we shall do. For convenience, we set $Y:=\mathbb{R}^{2} \otimes W^{1,(2,1)}$ and $Y_{0}:=\mathbb{R}^{2} \otimes W_{0}^{1,(2,1)}$. Let $g$ be as in (3.11), and $u$ be an arbitrary test function in $\mathbb{R}^{2} \otimes \mathcal{D}$. Clearly, $\vec{\Phi} u$ and $\vec{\Phi}_{k} u$ are elements of $Y_{0}$ with an estimate analogous to (3.4). Note that

$$
\left\langle\vec{\Phi}_{k} \nabla^{\perp} \vec{L}_{k}-\vec{\Phi} g, u\right\rangle_{\mathcal{D}^{\prime}, \mathcal{D}} \equiv\left\langle\nabla^{\perp} \vec{L}_{k}, u\left(\vec{\Phi}_{k}-\vec{\Phi}\right)\right\rangle_{Y_{0}^{\prime}, Y_{0}}+\left\langle\nabla^{\perp} \vec{L}_{k}-g, u \vec{\Phi}\right\rangle_{Y_{0}^{\prime}, Y_{0}}
$$

Whence, from the uniform boundedness of $\left(\nabla^{\perp} \vec{L}_{k}\right)$ in $\mathbb{R}^{2} \otimes W^{-1,(2, \infty)}$ and the convergences $(3.11)$ to $(3.12)$, the announced result follows.

As explained in Theorem 3.3.8 from [14], the fact that ${ }^{7}$

$$
\Delta \lambda_{k}=-\mathrm{e}^{2 \lambda_{k}} K_{k}
$$

is an element of the Hardy space $\mathcal{H}^{1}$ implies that $\left(\lambda_{k}\right)$ is a sequence of elements in $W^{1,(2,1)}$, uniformly bounded in norm by a constant depending only upon the uniform bound on $\left(\left\|\vec{\Phi}_{k}\right\|_{W^{2,2} \cap W^{1, \infty}}\right)$. Owing to the RellichKondrachov theorem, we may extract a subsequence $\left(\lambda_{k}\right)$ satisfying

$$
\lambda_{k} \longrightarrow \lambda \quad \text { in } \bigcap_{p<\infty} L^{p}
$$

for some $\lambda$. Furthermore, since $\lambda_{k} \simeq \log \left|\nabla \vec{\Phi}_{k}\right|$, we note from hypothesis (2.3) that the sequence $\left(\lambda_{k}\right)$ is uniformly bounded in $L^{\infty}$, from which it follows that $\lambda$ itself must be bounded.

Recall that for $j \in\{1,2\}$, there holds

$$
\mathrm{e}^{2 \lambda_{k}}=\left|\partial_{x_{j}} \vec{\Phi}_{k}\right|^{2} \quad \text { and thus } \quad \partial_{x_{i}}\left(\mathrm{e}^{2 \lambda_{k}}\right)=2 \partial_{x_{j}} \vec{\Phi}_{k} \cdot \partial_{x_{j} x_{i}} \vec{\Phi}_{k}
$$

Accordingly, from (2.3), the sequence $\left(\mathrm{e}^{2 \lambda_{k}}\right)$ is uniformly bounded in $W^{1,2} \cap$ $L^{\infty}$. Paired to the uniform boundedness of $\lambda_{k}$, this implies the same is true about the sequences $\left(\mathrm{e}^{ \pm \lambda_{k}}\right)$. Combining this to the convergences (3.12) and (3.13), we find

$$
\left\{\begin{array}{c}
\mathrm{e}^{ \pm \lambda_{k}} \longrightarrow \mathrm{e}^{ \pm \lambda} \\
\left(\vec{e}_{j}\right)_{k}:=\mathrm{e}^{-\lambda_{k}} \partial_{x_{j}} \vec{\Phi}_{k} \longrightarrow \mathrm{e}^{-\lambda} \partial_{x_{j}} \vec{\Phi}=: \vec{e}_{j} \quad \text { in } \bigcap_{p<\infty} L^{p} .
\end{array}\right.
$$

This argument sheds light onto the imposed assumption that $\left(\lambda_{k}\right)$ be a uniformly bounded sequence. It enables us to conclude that $\lambda$ lies in $L^{\infty}$,

${ }^{7}$ where $K_{k}$ denotes the Gaussian curvature of the immersion $\vec{\Phi}_{k}$. 
and thus in particular that $\mathrm{e}^{\lambda}$ is bounded from below; thereby making the limit $\vec{\Phi}$ into a non-degenerate immersion without collapse.

Because the sequence $\left(\vec{H}_{k}\right)$ is uniformly bounded in $L^{2}$, we can extract a weakly convergent subsequence:

$$
\vec{H}_{k} \longrightarrow \vec{H} \quad \text { in } \quad L^{2} \text {. }
$$

Altogether, (3.14) and (3.15) show that

$$
\left(\vec{e}_{1}\right)_{k} \wedge\left(\vec{e}_{2}\right)_{k} \wedge \vec{H}_{k} \longrightarrow \vec{e}_{1} \wedge \vec{e}_{2} \wedge \vec{H} \quad \text { in } \quad \bigcap_{1 \leq p<2} L^{p} .
$$

Equation (2.42) established in [24] states that

$$
\star\left(\vec{n}_{k}\left\llcorner\vec{H}_{k}\right)=(-1)^{m-1}\left(\vec{e}_{1}\right)_{k} \wedge\left(\vec{e}_{2}\right)_{k} \wedge \vec{H}_{k} .\right.
$$

Consequently, (3.16) is tantamount to

$$
\star\left(\vec { n } _ { k } \llcorner \vec { H } _ { k } ) \longrightarrow \star \left(\vec{n}\llcorner\vec{H}) \quad \text { in } \quad \bigcap_{1 \leq p<2} L^{p} .\right.\right.
$$

Combining this to (3.12) shows that

$$
\star\left(\vec { n } _ { k } \llcorner \vec { H } _ { k } ) \left\llcorner\nabla ^ { \perp } \vec { \Phi } _ { k } \longrightarrow \star \left(\vec { n } \llcorner \vec { H } ) \left\llcorner\nabla^{\perp} \vec{\Phi} \quad \text { in } \quad \bigcap_{1 \leq p<2} L^{p}\right.\right.\right.\right. \text {. }
$$

It takes little effort to verify that

$$
\operatorname{div}\left(u\left\llcorner\nabla^{\perp} v\right)=\nabla u\left\llcorner\nabla^{\perp} v\right.\right.
$$

holds in general. In particular, (3.17) yields

$$
\nabla\left(\star ( \vec { n } _ { k } \llcorner \vec { H } _ { k } ) ) \left\llcorner\nabla ^ { \perp } \vec { \Phi } _ { k } \longrightarrow \nabla \left(\star ( \vec { n } \llcorner \vec { H } ) ) \left\llcorner\nabla^{\perp} \vec{\Phi} \quad \text { in } \quad \mathcal{D}^{\prime}\left(D^{2}\right) .\right.\right.\right.\right.
$$

Per identity (2.6), the latter is equivalent to

$$
\nabla \vec{\Phi}_{k} \wedge \nabla \vec{H}_{k} \longrightarrow \nabla \vec{\Phi} \wedge \nabla \vec{H} \quad \text { in } \quad \mathcal{D}^{\prime}\left(D^{2}\right) .
$$

Finally, bringing altogether Lemmas 3.3, 3.4 and (3.18), we conclude as desired that, in the sense of distributions,

$$
\left\{\begin{array}{l}
\nabla \vec{\Phi} \cdot \nabla^{\perp} \vec{L}=0 \\
\nabla \vec{\Phi} \wedge \nabla^{\perp} \vec{L}=-2 \nabla \vec{\Phi} \wedge \nabla \vec{H}
\end{array}\right.
$$




\subsection{Proof of Theorem 2.2}

Since $\Delta \vec{\Phi} \wedge H=2 \mathrm{e}^{2 \lambda} \vec{H} \wedge \vec{H}=\overrightarrow{0}$, Equations (3.19) are in divergence form. In particular, we may introduce a scalar function $S$ and an $\mathbb{R}^{m}$-valued twoform $\vec{R}$ satisfying

$$
\left\{\begin{array}{l}
\nabla S:=\nabla \vec{\Phi} \cdot \vec{L}, \\
\nabla \vec{R}:=\nabla \vec{\Phi} \wedge \vec{L}+2 \nabla^{\perp} \vec{\Phi} \wedge \vec{H} .
\end{array}\right.
$$

Clearly, $S$ and $\vec{R}$ are defined on $D^{2}$ up to unimportant additive constants.

By hypothesis, $\vec{L}$ is an element of $L^{2, \infty}$, while $\vec{\Phi}$ is Lipschitz. Accordingly, $\nabla S$ and $\nabla \vec{R}$ belong to $L^{2, \infty}\left(D^{2}\right)$ and to $\mathbb{R}^{2} \otimes L^{2, \infty}\left(D^{2}\right)$, respectively. This observation and the particular structure of the right-hand side of the system (3.20) will enable us to deduce the regularity result we are seeking to obtain.

3.2.1. Regularity It is shown in $[24]^{8}$ that $S$ and $\vec{R}$ satisfy the system

$$
\left\{\begin{array}{l}
\Delta S=\nabla(\star \vec{n}) \cdot \nabla^{\perp} \vec{R}, \\
\Delta \vec{R}=-\nabla(\star \vec{n}) \bullet \nabla^{\perp} \vec{R}-\nabla(\star \vec{n}) \cdot \nabla^{\perp} S .
\end{array}\right.
$$

The advantage of these equations lies essentially in their right-hand sides comprising Jacobians. This peculiar feature will enable us to apply techniques of integration by compensation, in particular Wente-type estimates, which we now recall. They are due in parts to contributions by Wente [33], Tartar [28], Coifman et al. [10], Bethuel [5] and Hélein [14].

Lemma 3.5. Let $\Omega$ be an open subset of $\mathbb{R}^{2}$ with $C^{2}$-boundary. Suppose that $a$ and $b$ are elements of $W^{1,2}(\Omega)$ and of $W^{1,(2, \infty)}(\Omega)$, respectively. If $u$ satisfies

$$
\left\{\begin{aligned}
\Delta u & =\nabla a \cdot \nabla^{\perp} b & & \text { in } \Omega, \\
u & =0 & & \text { on } \partial \Omega,
\end{aligned}\right.
$$

then $\nabla u$ belongs to the space $L^{2}(\Omega)$ with the estimate

$$
\|\nabla u\|_{L^{2}(\Omega)} \lesssim\|\nabla a\|_{L^{2}(\Omega)}\|\nabla b\|_{L^{2, \infty}(\Omega)},
$$

up to a multiplicative constant depending only on $\Omega$.

\footnotetext{
${ }^{8}$ the equations appearing in [24] are slightly different (although equivalent) to those given here. The system (3.21) is derived in [4].
} 
The interested reader will find the proof of this result and further variations on the same theme in [12]. A second result (Theorem 3.4.1 in [14]) which will be useful to us is the following.

Lemma 3.6. Let $\Omega$ be an open subset of $\mathbb{R}^{2}$ with $C^{1}$-boundary. Suppose that $a$ and $b$ are elements of $W^{1,2}(\Omega)$. If $u$ satisfies

$$
\left\{\begin{aligned}
\Delta u & =\nabla a \cdot \nabla^{\perp} b & & \text { in } \Omega, \\
u & =0 & & \text { on } \partial \Omega,
\end{aligned}\right.
$$

then $u$ belongs to the space $W^{1,(2,1)}(\Omega) \subset C^{0}(\Omega)$ with the estimate

$$
\|\nabla u\|_{L^{2,1}(\Omega)} \lesssim\|\nabla a\|_{L^{2}(\Omega)}\|\nabla b\|_{L^{2}(\Omega)}
$$

up to a multiplicative constant depending only on $\Omega$.

Geared with these results, we are prepared to start our proof. Let us fix once and for all some point $x_{0} \in D_{1 / 2}(0)$ and some radius $0<r<1 / 2$, so that the flat disk $D_{r}\left(x_{0}\right)$ of radius $r$ and centered on the point $x_{0}$ is properly contained in the unit disc $D^{2}$. We use an argument developed by Bethuel [5]. With the help of the theorem of Fubini, we may always choose $r_{0} \in(r / 4, r / 2)$ such that

$$
\begin{aligned}
\int_{\partial D\left(x_{0}, r_{0}\right)}|\nabla S|^{\frac{3}{2}} & \lesssim \frac{1}{r} \int_{\partial D\left(x_{0}, r\right)}|\nabla S|^{\frac{3}{2}} \lesssim r^{-\frac{1}{2}}\|\nabla S\|_{L^{2, \infty}\left(D\left(x_{0}, r\right)\right)}^{\frac{3}{2}} \\
& \leq r^{-\frac{1}{2}}\|\nabla S\|_{L^{2, \infty}\left(D^{2}\right)}^{\frac{3}{2}}
\end{aligned}
$$

We define

$$
S=S_{0}+S_{1} \quad \text { and } \quad \vec{R}=\vec{R}_{0}+\vec{R}_{1}
$$

where the new variables, in accordance with (3.21), satisfy

$$
\left\{\begin{aligned}
\Delta S_{0}=0, & \Delta \vec{R}_{0}=\overrightarrow{0} \quad \text { in } \quad D_{r_{0}}\left(x_{0}\right), \\
S_{0}=S, & \vec{R}_{0}=\vec{R} \quad \text { on } \partial D_{r_{0}}\left(x_{0}\right),
\end{aligned}\right.
$$

and

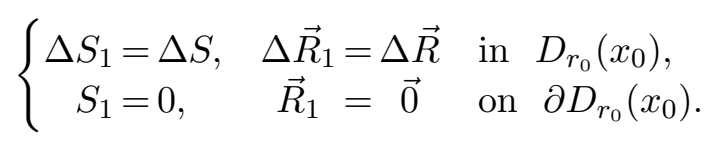


We set

$$
s:=\frac{1}{2 \pi r_{0}} \int_{\partial D_{r_{0}}\left(x_{0}\right)} S .
$$

Classical Sobolev embedding theorems then give

$$
\begin{aligned}
\left\|\nabla S_{0}\right\|_{L^{2}\left(D_{r_{0}}\left(x_{0}\right)\right)} & \leq\|S-s\|_{H^{1 / 2}\left(\partial D_{r_{0}}\left(x_{0}\right)\right)} \lesssim r^{\frac{1}{3}}\|\nabla S\|_{L^{3 / 2}\left(\partial D_{r_{0}}\left(x_{0}\right)\right)} \\
& \lesssim\|\nabla S\|_{L^{2, \infty}\left(D^{2}\right)}<\infty
\end{aligned}
$$

where (3.22) was used. In exactly the same manner, there holds

$$
\left\|\nabla \vec{R}_{0}\right\|_{L^{2}\left(D_{r_{0}}\left(x_{0}\right)\right)} \lesssim\|\nabla \vec{R}\|_{L^{2, \infty}\left(D^{2}\right)}<\infty
$$

Owing to (3.26) and (3.27), we may invoke classical growth estimates (cf., e.g., Theorem 3.3.12 in [14]) for the harmonic functions $S_{0}$ and $\vec{R}_{0}$, and so deduce that for $0<k<1$ one has:

$$
\left\|\nabla S_{0}\right\|_{L^{2}\left(D_{k r_{0}}\left(x_{0}\right)\right)} \lesssim k\left\|\nabla S_{0}\right\|_{L^{2}\left(D_{r_{0}}\left(x_{0}\right)\right)}
$$

and

$$
\left\|\nabla \vec{R}_{0}\right\|_{L^{2}\left(D_{k r_{0}}\left(x_{0}\right)\right)} \lesssim k\left\|\nabla \vec{R}_{0}\right\|_{L^{2}\left(D_{r_{0}}\left(x_{0}\right)\right)}
$$

We next move on to obtaining information for the functions $S_{1}$ and $\vec{R}_{1}$. Since the right-hand sides of (3.21), and thus of (3.25), comprise only Jacobians, Lemma 3.5 may be called upon so as to produce the estimates

$$
\left\{\begin{aligned}
\left\|\nabla S_{1}\right\|_{L^{2}\left(D_{r_{0}}\left(x_{0}\right)\right)} & \lesssim \varepsilon\|\nabla \vec{R}\|_{L^{2, \infty}\left(D_{r_{0}}\left(x_{0}\right)\right)}, \\
\left\|\nabla \vec{R}_{1}\right\|_{L^{2}\left(D_{r_{0}}\left(x_{0}\right)\right)} & \lesssim \varepsilon\left(\|\nabla S\|_{L^{2, \infty}\left(D_{r_{0}}\left(x_{0}\right)\right)}+\|\nabla \vec{R}\|_{L^{2, \infty}\left(D_{r_{0}}\left(x_{0}\right)\right)}\right)
\end{aligned}\right.
$$

up to some unimportant multiplicative constants. Here $\varepsilon$ denotes the (adjustable) upper bound on the energy:

$$
\|\nabla \vec{n}\|_{L^{2}\left(D^{2}\right)} \leq \varepsilon
$$

As by hypothesis $S$ and $\vec{R}$ are elements of $W^{1,(2, \infty)}$, the above estimates combined to (3.26) and (3.27) yield

$$
\|\nabla S\|_{L^{2}\left(D_{r_{0}}\left(x_{0}\right)\right)}+\|\nabla \vec{R}\|_{L^{2}\left(D_{r_{0}}\left(x_{0}\right)\right)}<\infty
$$


We are now in position of applying Lemma 3.6 to the system (3.25), thereby obtaining (since $k<1$ ),

$$
\left\{\begin{aligned}
&\left\|\nabla S_{1}\right\|_{L^{2,1}\left(D_{k r_{0}}\left(x_{0}\right)\right)} \lesssim \varepsilon\|\nabla \vec{R}\|_{L^{2}\left(D_{r_{0}}\left(x_{0}\right)\right)}, \\
&\left\|\nabla \vec{R}_{1}\right\|_{L^{2,1}\left(D_{k r_{0}}\left(x_{0}\right)\right)} \lesssim \varepsilon\left(\|\nabla S\|_{L^{2}\left(D_{r_{0}}\left(x_{0}\right)\right)}+\|\nabla \vec{R}\|_{L^{2}\left(D_{r_{0}}\left(x_{0}\right)\right)}\right)
\end{aligned}\right.
$$

In particular, since $L^{2,1} \subset L^{2}$ and $k<1$, the latter paired to (3.28) and (3.29) shows that for some positive constants $C_{0}$ and $C_{1}$, there holds

$$
E_{D_{k r_{0}}\left(x_{0}\right)}(S, \vec{R}) \leq\left(C_{0} k+C_{1} \varepsilon\right) E_{D_{r_{0}}\left(x_{0}\right)}(S, \vec{R})
$$

where, for notational convenience, we have set the Dirichlet energy

$$
E_{D_{\rho}\left(x_{0}\right)}(u, \vec{v}):=\|\nabla u\|_{L^{2}\left(D_{\rho}\left(x_{0}\right)\right)}+\|\nabla \vec{v}\|_{L^{2}\left(D_{\rho}\left(x_{0}\right)\right)}
$$

We have the freedom to adjust the positive parameter $\varepsilon$ as we please. Because $k \in(0,1)$, we may in particular arrange for the constant $\left(C_{0} k+C_{1} \varepsilon\right)$ to be smaller than 1. Then, iterating (3.31), we infer the existence of some $\gamma \in(0,1)$ such that

$$
E_{D_{\rho}(x)}(S, \vec{R}) \leq C \rho^{\gamma}
$$

holds for all $0<\rho<1 / 2$, all points $x \in D_{1 / 2}(0)$, and some constant $C>0$. With the help of the Poincaré inequality, the estimate (3.32) may be used to show that $S$ and $\vec{R}$ are locally Hölder continuous. We are however interested in another one of its corollaries. Consider the maximal function

$$
M_{2-\gamma} F(x):=\sup _{\rho>0} \rho^{-\gamma} \int_{D_{\rho}(x)}|F(y)| d y .
$$

Going back to (3.21), owing to (3.30) and (3.32), it follows that

$$
M_{2-\gamma}\left(\chi_{D_{1 / 2}(0)} \Delta S\right)(x) \leq \varepsilon \sup _{0<\rho<\frac{1}{2}} \rho^{-\gamma} E_{D_{\rho}(x)} \leq C \varepsilon
$$

is a uniformly bounded function on $D_{1 / 2}(0)$, and similarly with $\vec{R}$ in place of $S$. Here $\chi_{D_{1 / 2}(0)}$ denotes the characteristic function of the disc $D_{1 / 2}(0)$. It is clear that $\Delta S$ and $\Delta \vec{R}$ belong to $L^{1}\left(D_{1 / 2}(0)\right)$, since $S, \vec{R}$, and $\star \vec{n}$ are elements of $W^{1,2}\left(D_{1 / 2}(0)\right)$. We may thus call upon Proposition 3.2 in [2] to 
deduce that

$$
\frac{1}{|x|} * \chi_{D_{1 / 2}(0)} \Delta S \quad \text { and } \quad \frac{1}{|x|} * \chi_{D_{1 / 2}(0)} \Delta \vec{R}
$$

belong to $L^{q, \infty}\left(D_{1 / 2}(0)\right)$, with $q=\frac{2-\gamma}{1-\gamma}$.

A classical estimate about Riesz kernels states that in general, there holds

$$
|\nabla u|(y) \leq C_{1} \frac{1}{|x|} * \chi_{D_{1 / 2}(0)} \Delta u+C_{2}, \quad \forall y \in D_{1 / 4}(0),
$$

for two constants $C_{1}$ and $C_{2}$. We then infer that $\nabla S$ and $\nabla \vec{R}$ are elements of $L^{q, \infty}\left(D_{1 / 4}(0)\right)$, with $q$ as above. In particular, because $\gamma \in(0,1)$, it follows that $q>2$, and thus

$$
\nabla S, \nabla \vec{R} \in L^{2+\delta}\left(D_{1 / 4}(0)\right)
$$

for some $\delta>0$.

Now that we have this result at our disposal, we are ready to implement our regularity proof. It involves a bootstrapping argument, which will be performed on the following identity, established in Lemma A.4:

$$
-2 \Delta \vec{\Phi}=\nabla \vec{R} \bullet \nabla^{\perp} \vec{\Phi}+\nabla S \cdot \nabla^{\perp} \vec{\Phi} .
$$

By hypothesis, $\vec{\Phi}$ is a Lipschitz function. Combining (3.33) and the latter thus shows that

$$
\nabla \vec{\Phi} \in W^{1,2+\delta}\left(D_{1 / 4}(0)\right)
$$

Recall that

$$
\star \vec{n}=\vec{e}_{1} \wedge \vec{e}_{2} \quad \text { with } \quad \vec{e}_{j}:=\frac{\partial_{x_{j}} \vec{\Phi}}{\left|\partial_{x_{j}} \vec{\Phi}\right|} .
$$

Hence there holds

$$
\nabla \star \vec{n}=\frac{\sqrt{2}}{|\nabla \vec{\Phi}|}\left[\pi_{\vec{n}}\left(\partial_{x_{1}} \nabla \vec{\Phi}\right) \wedge \vec{e}_{2}+\vec{e}_{1} \wedge \pi_{\vec{n}}\left(\partial_{x_{2}} \nabla \vec{\Phi}\right)\right],
$$

and consequently

$$
|\nabla \vec{n}| \simeq|\nabla \vec{\Phi}|^{-1}\left|\pi_{\vec{n}} \nabla^{2} \vec{\Phi}\right| .
$$


Accordingly, (3.35) and the fact that $|\nabla \vec{\Phi}|$ is bounded from above and below yield that

$$
\nabla \vec{n} \in L^{2+\delta}\left(D_{1 / 4}(0)\right)
$$

For the reader's convenience, we recall

$$
\left\{\begin{array}{l}
\Delta S=\nabla(\star \vec{n}) \cdot \nabla^{\perp} \vec{R}, \\
\Delta \vec{R}=-\nabla(\star \vec{n}) \bullet \nabla^{\perp} \vec{R}-\nabla(\star \vec{n}) \cdot \nabla^{\perp} S .
\end{array}\right.
$$

Accounting for (3.33) and (3.37) in this system gives

$$
\nabla S, \nabla \vec{R} \in W^{1,1+\delta / 2} \subset L^{\beta} \quad \text { with } \quad \beta:=2 \frac{2+\delta}{2-\delta} .
$$

Comparing the latter to (3.33) reveals that the regularity has been improved.

We are dealing with a non-degenerate immersion $\vec{\Phi}$, so in particular the modulus $|\nabla \vec{\Phi}|$ is bounded from both above and below. Then (3.38) and (3.35) brought into (3.34) show that

$$
\nabla^{2} \vec{\Phi} \in W^{1, \beta}\left(D_{1 / 4}(0)\right)
$$

and hence

$$
|\nabla \vec{\Phi}|,|\nabla \vec{\Phi}|^{-1} \in W^{2, \beta}\left(D_{1 / 4}(0)\right) .
$$

Put into (3.36), the latter gives that $\nabla \vec{n} \in W^{1, \beta}$. The above procedure may then be repeated until we reach that all functions involved are continuous (from belonging to a Sobolev space of high enough order). Finally, a standard "strong solution analysis" of the equations eventually yields that $\vec{\Phi}$ is smooth, thereby concluding the proof.

3.2.2. Conformal Willmore equation We open our derivations by introducing some notation. Let $z=x_{1}+\mathrm{i} x_{2}$ and $\bar{z}$ be its complex conjugate. We set

$$
\vec{e}_{z}:=\mathrm{e}^{-\lambda} \partial_{z} \vec{\Phi}=\frac{1}{2}\left(\vec{e}_{1}-i \vec{e}_{2}\right) \quad \text { and } \quad \vec{e}_{\bar{z}}:=\mathrm{e}^{-\lambda} \partial_{\bar{z}} \vec{\Phi}=\frac{1}{2}\left(\vec{e}_{1}+i \vec{e}_{2}\right)
$$

Since $\vec{\Phi}$ is conformal, there holds

$$
\partial_{x_{j}} \vec{\Phi} \cdot \partial_{x_{k}} \vec{\Phi}=\mathrm{e}^{2 \lambda} \delta_{j k} \quad \text { for } \quad(j, k) \in\{1,2\}
$$

and thus

$$
\partial_{a} \vec{\Phi} \cdot \partial_{b} \vec{\Phi}=\frac{1}{2} \mathrm{e}^{2 \lambda} \delta_{a \bar{b}} \quad \text { for } \quad(a, b) \in\{z, \bar{z}\}
$$


From this, it follows easily that for any triple $(a, b, c) \in\{z, \bar{z}\}$ there holds

$$
\begin{aligned}
\partial_{a} \vec{\Phi} \cdot \partial_{b c} \vec{\Phi} & \equiv \delta_{c a}\left(\partial_{a} \vec{\Phi} \cdot \partial_{a b} \vec{\Phi}\right)+\delta_{c b}\left(\partial_{a} \vec{\Phi} \cdot \partial_{b b} \vec{\Phi}\right) \\
& =\frac{1}{2}\left(\delta_{c a}-\delta_{c b}\right) \partial_{b}\left|\partial_{a} \vec{\Phi}\right|^{2}+\delta_{c b} \partial_{b}\left(\partial_{a} \vec{\Phi} \cdot \partial_{b} \vec{\Phi}\right)=\delta_{c b} \delta_{a \bar{b}} \mathrm{e}^{2 \lambda} \partial_{b} \lambda
\end{aligned}
$$

Hence,

$$
\begin{aligned}
\vec{e}_{a} \cdot \partial_{b} \vec{e}_{c} & \equiv \mathrm{e}^{-2 \lambda}\left(\partial_{a} \vec{\Phi} \cdot \partial_{b c} \vec{\Phi}-\left(\partial_{b} \lambda\right) \partial_{a} \vec{\Phi} \cdot \partial_{c} \vec{\Phi}\right) \\
& =\frac{1}{2}\left(2 \delta_{c b} \delta_{a \bar{b}}-\delta_{a \bar{c}}\right) \partial_{b} \lambda
\end{aligned}
$$

Observe furthermore that

$$
\vec{e}_{a} \cdot \vec{e}_{b}=\frac{1}{2} \delta_{a \bar{b}}
$$

Thus, combining (3.39) and (3.40) gives

$$
\partial_{b} \vec{e}_{c}=\partial_{b} \lambda \sum_{a \in\{z, \bar{z}\}}\left(2 \delta_{c b} \delta_{a b}-\delta_{a c}\right) \vec{e}_{a}+\pi_{\vec{n}}\left(\partial_{b} \vec{e}_{c}\right)
$$

Next, we have

$$
\begin{aligned}
\vec{n}_{\alpha} \cdot \partial_{\bar{z}} \vec{e}_{z} & =-\vec{e}_{z} \cdot \partial_{\bar{z}} \vec{n}_{\alpha}=-\frac{1}{4}\left(\vec{e}_{1}-\mathrm{i} \vec{e}_{2}\right)\left(\partial_{x_{1}}+\mathrm{i} \partial_{x_{2}}\right) \vec{n}_{\alpha} \\
& =\frac{\mathrm{e}^{\lambda}}{4}\left(h_{11}^{\alpha}+h_{22}^{\alpha}\right)=\frac{\mathrm{e}^{\lambda}}{2} H^{\alpha}
\end{aligned}
$$

and similarly

$$
\vec{n}_{\alpha} \cdot \partial_{\bar{z}} \vec{e}_{\bar{z}}=\frac{\mathrm{e}^{\lambda}}{2} H_{0}^{\alpha} .
$$

Accordingly, we may now deduce from (3.41) that

$$
\left\{\begin{array}{l}
\partial_{\bar{z}} \vec{e}_{z}=-\left(\partial_{\bar{z}} \lambda\right) \vec{e}_{z}+\frac{\mathrm{e}^{\lambda}}{2} \vec{H} \\
\partial_{\bar{z}} \vec{e}_{\bar{z}}=\left(\partial_{\bar{z}} \lambda\right) \vec{e}_{\bar{z}}+\frac{\mathrm{e}^{\lambda}}{2} \vec{H}_{0}
\end{array}\right.
$$

Note that we can also easily obtain from the above computations the identity

$$
\partial_{\bar{z}} \vec{n}_{\alpha}=-\mathrm{e}^{\lambda}\left(H_{0}^{\alpha} \vec{e}_{z}+H^{\alpha} \vec{e}_{\bar{z}}\right)+\pi_{\vec{n}}\left(\partial_{\bar{z}} \vec{n}_{\alpha}\right) .
$$

These expressions shall come helpful in the sequel. 
Suppose now that $\vec{\Phi}$ is smooth and satisfies for some $\vec{L}$ the system

$$
\left\{\begin{array}{l}
\nabla \vec{\Phi} \cdot \nabla^{\perp} \vec{L}=0 \\
\nabla \vec{\Phi} \wedge \nabla^{\perp} \vec{L}=-2 \nabla \vec{\Phi} \wedge \nabla \vec{H}
\end{array}\right.
$$

Since $\vec{e}_{j}=\mathrm{e}^{-\lambda} \partial_{x_{j}} \vec{\Phi}$, the first equation in the system is equivalent to

$$
\vec{e}_{1} \cdot \partial_{x_{2}} \vec{L}=\vec{e}_{2} \cdot \partial_{x_{1}} \vec{L}
$$

Whence, we deduce that $\vec{L}$ satisfies

$$
\nabla^{\perp} \vec{L}=\left(\begin{array}{cc}
a & b \\
c & -a
\end{array}\right)\left(\begin{array}{c}
\vec{e}_{1} \\
\vec{e}_{2}
\end{array}\right)+\left(\begin{array}{c}
p_{1}^{\alpha} \\
p_{2}^{\alpha}
\end{array}\right) \vec{n}_{\alpha}
$$

for some suitable coefficients $a, b, c$ and $p_{j}^{\alpha}$.

Substituting this form in the second equation from (3.44) gives

$$
\begin{aligned}
& \vec{e}_{1} \wedge\left[2\left(\vec{e}_{1} \cdot \partial_{x_{1}} \vec{H}\right) \vec{e}_{1}+2\left(\vec{e}_{2} \cdot \partial_{x_{1}} \vec{H}\right) \vec{e}_{2}+2\left(\vec{n}_{\alpha} \cdot \partial_{x_{1}} \vec{H}\right) \vec{n}_{\alpha}\right. \\
& \left.\quad+a \vec{e}_{1}+b \vec{e}_{2}+p_{1}^{\alpha} \vec{n}_{\alpha}\right] \\
& \quad=-\vec{e}_{2} \wedge\left[2\left(\vec{e}_{1} \cdot \partial_{x_{2}} \vec{H}\right) \vec{e}_{1}+2\left(\vec{e}_{2} \cdot \partial_{x_{2}} \vec{H}\right) \vec{e}_{2}+2\left(\vec{n}_{\alpha} \cdot \partial_{x_{2}} \vec{H}\right) \vec{n}_{\alpha}\right. \\
& \left.\quad+c \vec{e}_{1}-a \vec{e}_{2}+p_{2}^{\alpha} \vec{n}_{\alpha}\right],
\end{aligned}
$$

thereby yielding

$$
c-b=2\left(\vec{e}_{2} \cdot \partial_{x_{1}}-\vec{e}_{1} \cdot \partial_{x_{2}}\right) \vec{H} \quad \text { and } \quad p_{j}^{\alpha}=-2 \vec{n}_{\alpha} \cdot \partial_{x_{j}} \vec{H}
$$

However, because $\vec{H}=H^{\alpha} \vec{n}_{\alpha}$ and the second fundamental form is symmetric, there holds

$$
\begin{aligned}
c-b & =2\left(\vec{e}_{2} \cdot \partial_{x_{1}}-\vec{e}_{1} \cdot \partial_{x_{2}}\right) \vec{H}=2 H^{\alpha}\left(\vec{e}_{1} \cdot \partial_{x_{2}}-\vec{e}_{2} \cdot \partial_{x_{1}}\right) \vec{n}_{\alpha} \\
& =2 H^{\alpha} \mathrm{e}^{\lambda}\left(h_{21}^{\alpha}-h_{12}^{\alpha}\right)=0 .
\end{aligned}
$$

Whence, (3.45) may be recast in the form

$$
\nabla^{\perp} \vec{L}=\left(\begin{array}{cc}
a & b \\
b & -a
\end{array}\right)\left(\begin{array}{c}
\vec{e}_{1} \\
\vec{e}_{2}
\end{array}\right)-2 \pi_{\vec{n}}(\nabla \vec{H}) .
$$

Equivalently, in the $\left\{\vec{e}_{z}, \vec{e}_{\bar{z}}\right\}$-frame, this expression reads

$$
\partial_{z} \vec{L}=A \vec{e}_{\bar{z}}-2 \mathrm{i} \pi_{\vec{n}}\left(\partial_{z} \vec{H}\right)
$$

where $A:=b+\mathrm{i} a$. 
Owing to the identities (3.42), there holds

$$
\begin{aligned}
\partial_{\bar{z}} \pi_{\vec{n}} \partial_{z} \vec{H}-\pi_{\vec{n}} \partial_{\bar{z}} \pi_{\vec{n}} \partial_{z} \vec{H} \\
\quad \equiv 2\left(\vec{e}_{z} \cdot \partial_{\bar{z}} \pi_{\vec{n}} \partial_{z} \vec{H}\right) \vec{e}_{\bar{z}}+2\left(\vec{e}_{\bar{z}} \cdot \partial_{\bar{z}} \pi_{\vec{n}} \partial_{z} \vec{H}\right) \vec{e}_{z} \\
\quad=-2\left(\partial_{\bar{z}} \vec{e}_{z} \cdot \pi_{\vec{n}} \partial_{z} \vec{H}\right) \vec{e}_{\bar{z}}-2\left(\partial_{\bar{z}} \vec{e}_{\bar{z}} \cdot \pi_{\vec{n}} \partial_{z} \vec{H}\right) \vec{e}_{z} \\
\quad=-\left(\mathrm{e}^{\lambda} \vec{H} \cdot \pi_{\vec{n}} \partial_{z} \vec{H}\right) \vec{e}_{\bar{z}}-\left(\mathrm{e}^{\lambda} \vec{H}_{0} \cdot \pi_{\vec{n}} \partial_{z} \vec{H}\right) \vec{e}_{z} \\
\quad=-\mathrm{e}^{\lambda}\left[\left(\vec{H} \cdot \partial_{z} \vec{H}\right) \vec{e}_{\bar{z}}+\left(\vec{H}_{0} \cdot \partial_{z} \vec{H}\right) \vec{e}_{z}\right] .
\end{aligned}
$$

From (3.46), the latter, and (3.43), we see that

$$
\begin{aligned}
\partial_{\bar{z} z} \vec{L}= & \partial_{\bar{z}}\left(A \vec{e}_{\bar{z}}\right)-2 \mathrm{i} \partial_{\bar{z}} \pi_{\vec{n}} \partial_{z} \vec{H} \\
= & \partial_{\bar{z}}\left(A \vec{e}_{\bar{z}}\right)+2 \mathrm{ie}^{\lambda}\left[\left(\vec{H} \cdot \partial_{z} \vec{H}\right) \vec{e}_{\bar{z}}+\left(\vec{H}_{0} \cdot \partial_{z} \vec{H}\right) \vec{e}_{z}\right] \\
& -2 \mathrm{i} \pi_{\vec{n}} \partial_{\bar{z}} \pi_{\vec{n}} \partial_{z} \vec{H} \\
= & \mathrm{e}^{-\lambda} \partial_{\bar{z}}\left(\mathrm{e}^{\lambda} A\right) \vec{e}_{\bar{z}}+2 \mathrm{ie}^{\lambda}\left[\left(\vec{H} \cdot \partial_{z} \vec{H}\right) \vec{e}_{\bar{z}}+\left(\vec{H}_{0} \cdot \partial_{z} \vec{H}\right) \vec{e}_{z}\right] \\
& +\frac{\mathrm{e}^{\lambda}}{2} A \vec{H}_{0}-2 \mathrm{i} \pi_{\vec{n}} \partial_{\bar{z}} \pi_{\vec{n}} \partial_{z} \vec{H}
\end{aligned}
$$

Because the imaginary part $\Im\left(\partial_{\bar{z} z} \vec{L}\right)=0$, we separate this last identity into its normal and tangential components to discover

$$
\partial_{\bar{z}}\left(\mathrm{e}^{\lambda} A\right)=-2 \mathrm{i}^{2 \lambda}\left(\vec{H} \cdot \partial_{z} \vec{H}+\overline{\vec{H}_{0}} \cdot \partial_{\bar{z}} \vec{H}\right)
$$

and

$$
4 \Im\left[\mathrm{i} \pi_{\vec{n}} \partial_{\bar{z}} \pi_{\vec{n}} \partial_{z} \vec{H}\right]=\mathrm{e}^{\lambda} \Im\left(A \vec{H}_{0}\right) .
$$

Comparing (3.49) to the Codazzi-Mainardi equation (A.21) shows that

$$
\partial_{\bar{z}}\left[\mathrm{e}^{\lambda}\left(A+2 \mathrm{i}^{\lambda} \overline{\vec{H}_{0}} \cdot \vec{H}\right)\right]=0 .
$$

Accordingly, there exists some holomorphic function $f(z)$ such that

$$
A=-\mathrm{e}^{-\lambda} f(z)-2 \mathrm{i} \mathrm{e}^{\lambda} \overline{\vec{H}_{0}} \cdot \vec{H}
$$

In conformal coordinates, there holds

$$
\mathrm{e}^{2 \lambda} \Delta_{\perp} \vec{H}=\pi_{\vec{n}}\left(\operatorname{div} \pi_{\vec{n}}(\nabla \vec{H})\right) \equiv 4 \Im\left[\mathrm{i} \pi_{\vec{n}} \partial_{\bar{z}} \pi_{\vec{n}} \partial_{z} \vec{H}\right]
$$

so that (3.50) becomes

$$
\mathrm{e}^{\lambda} \Delta_{\perp} \vec{H}=\Im\left(A \vec{H}_{0}\right) .
$$


Introducing (3.51) in the latter gives thus

$$
\Delta_{\perp} \vec{H}+2 \Re\left(\left(\overrightarrow{\vec{H}_{0}} \cdot \vec{H}\right) \vec{H}_{0}\right)=\mathrm{e}^{-2 \lambda} \Im\left(\vec{H}_{0} f\right) .
$$

To complete our derivation, there remains to observe that

$$
\begin{aligned}
\Re\left[H_{0}^{\alpha} \overline{H_{0}^{\beta}}\right] & =\frac{1}{4}\left[\left(h_{11}^{\alpha}-h_{22}^{\alpha}\right)\left(h_{11}^{\beta}-h_{22}^{\beta}\right)+4 h_{12}^{\alpha} h_{12}^{\beta}\right] \\
& =\frac{1}{2} \sum_{i, j=1}^{2} h_{i j}^{\alpha} h_{i j}^{\beta}-\frac{1}{4}\left(h_{11}^{\alpha}+h_{22}^{\alpha}\right)\left(h_{11}^{\beta}+h_{22}^{\beta}\right) \\
& =\frac{1}{2} \sum_{i, j=1}^{2} h_{i j}^{\alpha} h_{i j}^{\beta}-H^{\alpha} H^{\beta} .
\end{aligned}
$$

Accordingly, (3.52) yields

$$
\Delta_{\perp} \vec{H}+\sum_{\alpha, \beta=1}^{m-2} \sum_{i, j=1}^{2} h_{i j}^{\alpha} h_{i j}^{\beta} H^{\beta} \vec{n}_{\alpha}-2|\vec{H}|^{2} \vec{H}=\mathrm{e}^{-2 \lambda} \Im\left(\vec{H}_{0} f\right) .
$$

This identity is the desired conformal Willmore equation announced in Theorem 2.2.

The converse of Theorem 2.2 is easily deduced from reversing the above computations. Assuming that (3.53) holds for some holomorphic function $f$, we may define $A$ as in (3.51). The fact that $f$ is holomorphic, along with our previous derivations, notably (3.47) and (3.48), yield

$$
\begin{aligned}
\partial_{\bar{z}}\left(A \vec{e}_{\bar{z}}-2 \mathrm{i} \pi_{\vec{n}} \partial_{z} \vec{H}\right)= & \mathrm{e}^{-\lambda} \partial_{\bar{z}}\left(\mathrm{e}^{\lambda} A\right) \vec{e}_{\bar{z}}+\frac{\mathrm{e}^{\lambda}}{2} A \vec{H}_{0}+2 \mathrm{i} \pi_{\vec{n}} \partial_{\bar{z}} \pi_{\vec{n}} \partial_{z} \vec{H} \\
& -2 \mathrm{i}^{\lambda}\left(\left(\vec{H} \cdot \partial_{z} \vec{H}\right) \vec{e}_{\bar{z}}+\left(\vec{H}_{0} \cdot \partial_{z} \vec{H}\right) \vec{e}_{z}\right) \\
= & -2 \mathrm{i}^{-\lambda} \partial_{\bar{z}}\left(\mathrm{e}^{2 \lambda} \overrightarrow{\vec{H}_{0}} \cdot \vec{H}\right) \vec{e}_{\bar{z}}+\frac{1}{2} f \vec{H}_{0} \\
& -i \mathrm{e}^{2 \lambda}\left(\overrightarrow{\vec{H}_{0}} \cdot \vec{H}\right) \vec{H}_{0}+2 \mathrm{i} \pi_{\vec{n}} \partial_{\bar{z}} \pi_{\vec{n}} \partial_{z} \vec{H} \\
& -2 \mathrm{i} \mathrm{e}^{\lambda}\left(\left(\vec{H} \cdot \partial_{z} \vec{H}\right) \vec{e}_{\bar{z}}+\left(\vec{H}_{0} \cdot \partial_{z} \vec{H}\right) \vec{e}_{z}\right) .
\end{aligned}
$$

Taking the imaginary part on both sides of the latter, and introducing the conformal Willmore equation (3.53) and the Codazzi-Mainardi equation (A.21), we find

$$
\Im\left[\partial_{\bar{z}}\left(A \vec{e}_{\bar{z}}-2 i \pi_{\vec{n}} \partial_{z} \vec{H}\right)\right]=0 .
$$


On the contractible unit disc, we deduce that there exists some $\vec{L}$ such that

$$
\partial_{z} \vec{L}=A \vec{e}_{\bar{z}}-2 \mathrm{i} \pi_{\vec{n}} \partial_{z} \vec{H}
$$

or equivalently

$$
\nabla^{\perp} \vec{L}=\mathrm{e}^{-\lambda}\left(\begin{array}{cc}
\Im(A) & \Re(A) \\
\Re(A) & -\Im(A)
\end{array}\right) \nabla \vec{\Phi}-2 \pi_{\vec{n}}(\nabla \vec{H})
$$

It takes little effort to verify ${ }^{9}$ that

$$
\nabla \vec{\Phi} \cdot \nabla^{\perp} \vec{L}=0
$$

which is the first part of the system (2.5). Similarly, calling upon the identity (A.17), one finally finds the desired

$$
\nabla \vec{\Phi} \wedge \nabla^{\perp} \vec{L}=-2 \nabla \vec{\Phi} \wedge \pi_{\vec{n}}(\nabla \vec{H})=-2 \nabla \vec{\Phi} \wedge \nabla \vec{H}
$$

\section{A. Appendix}

\section{A.1. Notational Conventions}

We append an arrow to all the elements belonging to $\mathbb{R}^{m}$. To simplify the notation, by $\vec{\Phi} \in X\left(D^{2}\right)$ is meant $\vec{\Phi} \in X\left(D^{2}, \mathbb{R}^{m}\right)$ whenever $X$ is a function space. Similarly, we write $\nabla \vec{\Phi} \in \mathbb{R}^{2} \otimes X\left(D^{2}\right)$ for $\nabla \vec{\Phi} \in X\left(D^{2}, \mathbb{R}^{2 m}\right)$.

Although this custom may seem at first odd, we allow the differential operators classically acting on scalars to act on elements of $\mathbb{R}^{m}$. Thus, for example, $\nabla \vec{\Phi}$ is the element of $\left(\partial_{x_{1}} \vec{\Phi}, \partial_{x_{2}} \vec{\Phi}\right) \in \mathbb{R}^{2} \otimes \mathbb{R}^{m}$. If $S$ is a scalar and $\vec{R}$ an element of $\mathbb{R}^{m}$, then we let

$$
\begin{aligned}
\vec{R} \cdot \nabla \vec{\Phi} & :=\left(\vec{R} \cdot \partial_{x_{1}} \vec{\Phi}, \vec{R} \cdot \partial_{x_{2}} \vec{\Phi}\right) \\
\nabla^{\perp} S \cdot \nabla \vec{\Phi} & :=\partial_{x_{1}} S \partial_{x_{2}} \vec{\Phi}-\partial_{x_{2}} S \partial_{x_{1}} \vec{\Phi} \\
\nabla^{\perp} \vec{R} \cdot \nabla \vec{\Phi} & :=\partial_{x_{1}} \vec{R} \cdot \partial_{x_{2}} \vec{\Phi}-\partial_{x_{2}} \vec{R} \cdot \partial_{x_{1}} \vec{\Phi} \\
\nabla^{\perp} \vec{R} \wedge \nabla \vec{\Phi} & :=\partial_{x_{1}} \vec{R} \wedge \partial_{x_{2}} \vec{\Phi}-\partial_{x_{2}} \vec{R} \wedge \partial_{x_{1}} \vec{\Phi}
\end{aligned}
$$

Similar quantities are defined following the same logic.

\footnotetext{
${ }^{9}$ recall that the operator $\nabla \vec{\Phi} \cdot \pi_{\vec{n}}$ is trivial.
} 
Two operations between multivectors are useful. The interior multiplication $L$ maps a pair comprising a $q$-vector $\gamma$ and a $p$-vector $\beta$ to a $(q-p)$ vector. It is defined via

$$
\langle\gamma\llcorner\beta, \alpha\rangle=\langle\gamma, \beta \wedge \alpha\rangle \quad \text { for each }(q-p) \text {-vector } \alpha \text {. }
$$

Let $\alpha$ be a $k$-vector. The first-order contraction operation $\bullet$ is defined inductively through

$$
\alpha \bullet \beta=\alpha\llcorner\beta \quad \text { when } \beta \text { is a 1-vector, }
$$

and

$$
\alpha \bullet(\beta \wedge \gamma)=(\alpha \bullet \beta) \wedge \gamma+(-1)^{p q}(\alpha \bullet \gamma) \wedge \beta,
$$

when $\beta$ and $\gamma$ are, respectively, a $p$-vector and a $q$-vector.

\section{A.2. Lorentz and Sobolev-Lorentz Spaces}

For the reader's convenience, we recall in this section the fundamentals of Lorentz spaces. Detailed accounts may be found in [3, 17, 27].

For a real-valued measurable function $f$ on an open subset of $U \subset \mathbb{R}^{n}$, its belonging to a Lorentz space is determined by a condition involving the non-decreasing rearrangement of $|f|$ on the interval $(0,|U|)$, where $|U|$ denotes the Lebesgue measure of $U$. The non-increasing rearrangement $f^{*}$ of $|f|$ is the unique positive continuous function from $(0,|U|)$ into $\mathbb{R}$ which is non-increasing and satisfies

$$
|\{x \in U|| f(x) \mid \geq s\}|=\left|\left\{t \in(0,|U|) \mid f^{*}(t) \geq s\right\}\right|
$$

If $p \in(1, \infty)$ and $q \in[1, \infty]$, the Lorentz space $L^{p, q}(U)$ is the set of measurable functions $f: U \rightarrow \mathbb{R}$ for which

$$
\int_{0}^{\infty}\left(t^{1 / p} f^{*}(t)\right)^{q} \frac{d t}{t}<\infty \quad \text { if } q<\infty
$$

or

$$
\sup _{t>0} t^{1 / p} f^{*}(t)<\infty \quad \text { if } q=\infty .
$$

A complete norm on $L^{p, q}(U)$ is given by

$$
\|f\|_{L^{p, q}(U)}=\left\|t^{1 / p} f^{* *}\right\|_{L^{q}([0, \infty), d t / t)} \quad \text { where } \quad f^{* *}(t):=\frac{1}{t} \int_{0}^{t} f^{*}(\tau) d \tau .
$$


One verifies that

$$
L^{p}=L^{p, p}
$$

and that $L^{p, \infty}$ is the weak- $L^{p}$ Marcinkiewicz space.

Moreover, we have the inclusions

$$
L^{p, 1} \subset L^{p, q^{\prime}} \subset L^{p, q^{\prime \prime}} \subset L^{p, \infty} \quad \text { for } \quad 1<q^{\prime}<q^{\prime \prime}<\infty ;
$$

and if $U$ has finite measure, there holds for all $q$ and $q^{\prime}$

$$
L^{p^{\prime}, q^{\prime}}(U) \subset L^{p, q}(U) \quad \text { whenever } \quad p<p^{\prime} .
$$

More precisely,

$$
\|f\|_{L^{p, q}(U)} \lesssim|U|^{\frac{p^{\prime}-p}{p^{\prime} p}}\|f\|_{L^{p^{\prime}, q^{\prime}}(U)}
$$

Finally, if $q<\infty$, the space $L^{\frac{p}{p-1}, \frac{q}{q-1}}$ is the dual of $L^{p, q}$.

Similarly to Lebesgue spaces, Lorentz spaces obey a pointwise multiplication rule and a convolution product rule. More precisely, for $1<p_{1}, p_{2}<\infty$ and $1 \leq q_{1}, q_{2} \leq \infty$, there holds

$$
L^{p_{1}, q_{1}} \times L^{p_{2}, q_{2}} \subset L^{p, q} \quad \text { with } \quad\left\{\begin{array}{l}
p^{-1}=p_{1}^{-1}+p_{2}^{-1}, \\
q^{-1}=q_{1}^{-1}+q_{2}^{-1}
\end{array}\right.
$$

and

$$
L^{p_{1}, q_{1}} * L^{p_{2}, q_{2}} \subset L^{p, q} \quad \text { with } \quad\left\{\begin{array}{l}
p^{-1}=p_{1}^{-1}+p_{2}^{-1}-1 \\
q^{-1}=q_{1}^{-1}+q_{2}^{-1}
\end{array}\right.
$$

An interesting feature of Lorentz spaces is that they are interpolation spaces between Lebesgue spaces.

Lorentz spaces offer the possibility to sharpen the classical Sobolev embedding theorem. More precisely, it can be shown (see [3, 29]) that

$$
W^{k, q}\left(\mathbb{R}^{m}\right) \subset L^{p, q}\left(\mathbb{R}^{m}\right)
$$

is a continuous embedding so long as

$$
1 \leq q \leq p<\infty \quad \text { and } \quad \frac{k}{m}=\frac{1}{q}-\frac{1}{p} .
$$

Our study requires that we introduce Sobolev-Lorentz spaces. These spaces are defined analogously to the "standard" Sobolev spaces, but with 
the Lebesgue norms replaced by Lorentz norms. In an effort to simplify the presentation, we shall focus only on the two-dimensional unit disc $D^{2}$. Let $m \in \mathbb{N}, p \in(1, \infty)$, and $q \in[1, \infty]$. The (homogeneous) Sobolev-Lorentz space $W^{m,(p, q)}\left(D^{2}\right)$ consists of all locally summable functions $u$ on $D^{2}$ such that $D^{\alpha} u$ exists in the weak sense and belongs to the Lorentz space $L^{p, q}\left(D^{2}\right)$ for each multiindex $\alpha$ with $|\alpha|=m$. The norm

$$
\|u\|_{W^{m,(p, q)}}:=\sum_{|\alpha|=m}\left\|D^{\alpha} u\right\|_{L^{p, q}}
$$

clearly makes $W^{m,(p, q)}\left(D^{2}\right)$ into a Banach space. The space $W^{0,(p, q)}$ is understood to be $L^{p, q}$. For notational convenience, we shall from now on omit to precise that we work on the unit disc $D^{2}$. This shall arise no confusion. We also focus on a case of particular interest to us, namely $(p, q)=(2,1)$. Because $L^{2,1}$ is a subspace of $L^{2}$, it follows immediately that for all $m \in \mathbb{N}$ there holds

$$
W^{m,(2,1)} \subset W^{m, 2}
$$

where $W^{m, 2}$ is the usual (homogeneous) Sobolev space. The embedding (A.2) shows that each element of $W^{m,(2,1)}$ has a well-defined trace on the boundary of the unit disc, for $m \geq 1$. If that trace is null, the said element belongs to the space $W_{0}^{m,(2,1)}$.

In [7], the authors prove ${ }^{10}$ that $W_{0}^{1,(2,1)}$ is a subspace of $L^{\infty} \cap C^{0}$, so that $W_{0}^{m,(2,1)}$ is a subspace of $W^{m-1, \infty} \cap C^{m-1}$. Altogether, there holds

$$
W_{0}^{m,(2,1)} \subset W^{m, 2} \cap W^{m-1, \infty} \cap C^{m-1}
$$

The Rellich-Kondrachov theorem states that $W^{1,2}$ is compact in $L^{r}$ for all finite $r \geq 1$. By standard interpolation techniques, it follows that

$$
W^{m, 2} \subset \subset W^{m-1,(p, q)} \quad \text { for } \quad 1<p<\infty, 1 \leq q \leq \infty
$$

is a compact inclusion for every $m \in \mathbb{N}^{*}$.

It is instructive to characterize the dual space of $W_{0}^{m,(p, q)}$, denoted $W^{-m,\left(p^{\prime}, q^{\prime}\right)}$, where $\left(p^{\prime}, q^{\prime}\right)$ is the conjugate pair of $(p, q)$ :

$$
p^{\prime}=\left(1-p^{-1}\right)^{-1} \quad \text { and } \quad q^{\prime}=\left(1-q^{-1}\right)^{-1} \text {. }
$$

\footnotetext{
${ }^{10}$ see also Theorem 3.3.4 in [14].
} 
Proposition A.1. Let $T$ be an element of $W^{-m,\left(p^{\prime}, q^{\prime}\right)}\left(D^{2}, \mathbb{R}\right)$. For every $\delta>0$, there exists an element $P$ of $W^{1-m,\left(p^{\prime}, q^{\prime}\right)}\left(D^{2}, \mathbb{R}^{2}\right)$ such that

$$
T=\operatorname{div} P,
$$

and

$$
\|P\|_{W^{1-m,\left(p^{\prime}, q^{\prime}\right)}} \leq\|T\|_{W^{-m,\left(p^{\prime}, q^{\prime}\right)}}+\delta .
$$

Proof. This is a mere adaption of the analogous statement for the LebesgueSobolev space $W^{m, p}$, ultimately following from the Hahn-Banach theorem. The reader is referred to Theorem 3.10 in [1] for details.

We next bring into light an interesting Hodge decomposition result which follows from standard elliptic theory and the interpolation nature of Lorentz spaces (cf. Proposition 3.3.9 in [14]).

Proposition A.2. To every vector field $\vec{g} \in L^{1}\left(D^{2}, \mathbb{R}^{2}\right)$ we associate the functions $\alpha, \beta$, and $h$ on $D^{2}$ which are solutions of

$$
\left\{\begin{array}{lll}
\Delta \alpha=\operatorname{div} \vec{g}, & \Delta \beta=\operatorname{curl} \vec{g} & \text { in } D^{2}, \\
\alpha=0, & \beta=0 & \text { on } \partial D^{2},
\end{array}\right.
$$

with

$$
\vec{g}=\nabla \alpha+\nabla^{\perp} \beta+\nabla h
$$

so that $h$ is harmonic on $D^{2}$.

Then the operators

$$
\vec{g} \longmapsto \nabla \alpha, \quad \vec{g} \longmapsto \nabla \beta, \quad \text { and } \quad \vec{g} \longmapsto \nabla h
$$

map continuously $L^{p, q}\left(D^{2}, \mathbb{R}^{2}\right)$ into itself for every choice of $p \in(1, \infty)$ and $q \in[1, \infty]$.

This lemma is the central ingredient of the following result. 
Lemma A.1. Suppose that $G=G_{1}+G_{2}+G_{3}$ satisfies

$$
\operatorname{div} G=0 \quad \text { in } \quad D^{2}
$$

where

$$
G_{1} \in W^{-1,(2, \infty)}\left(D^{2}, \mathbb{R}^{2}\right), \quad G_{2} \in W^{-1,2}\left(D^{2}, \mathbb{R}^{2}\right), \quad G_{3} \in L^{1}\left(D^{2}, \mathbb{R}^{2}\right)
$$

Then there exists an element $L$ in the space $L^{2, \infty}\left(D^{2}, \mathbb{R}\right)$ such that

$$
G=\nabla^{\perp} L
$$

Proof. A classical result of Laurent Schwartz guarantees the existence of $L_{0}$ in $L^{1}$ such that $G=\nabla^{\perp} L_{0}$ holds in the sense of distributions. Owing to the density of $C_{c}^{\infty}$ in $W_{0}^{1,(2,1)}$, it follows that

$$
\int_{D^{2}} G \cdot F=-\int_{D^{2}} L_{0} \operatorname{curl} F, \quad \forall F \in W_{0}^{1,(2,1)}\left(D^{2}, \mathbb{R}^{2}\right) .
$$

Next, let $f$ be an arbitrary element of $L^{2,1}\left(D^{2}, \mathbb{R}\right)$. Choosing

$$
\vec{g}=\left(0, f-\int_{D^{2}} f\right)
$$

in Proposition A.2, we infer the existence of an element $F:=(-\alpha, \beta)$ in $W_{0}^{1,(2,1)}\left(D^{2}, \mathbb{R}^{2}\right)$ satisfying

$$
\operatorname{curl} F=f-\int_{D^{2}} f
$$

as well as

$$
\|F\|_{W^{1,(2,1)}} \lesssim\|f\|_{L^{2,1}}
$$

Putting (A.10) into (A.9) then yields

$$
-\int_{D^{2}} L f=\int_{D^{2}} G \cdot F, \quad \text { where } \quad L:=L_{0}-\int_{D^{2}} L_{0} .
$$

Clearly, the function $L$ is a solution to (A.8). Recall that

$$
W_{0}^{1,(2,1)} \subset W_{0}^{1,2} \cap L^{\infty} \quad \text { and } \quad W^{-1,(2, \infty)}=\left(W_{0}^{1,(2,1)}\right)^{\prime},
$$


Accordingly, using (A.12) and (A.11), we find

$$
\begin{aligned}
\left|\int_{D^{2}} L f\right| & \leq\left|\int_{D^{2}} G_{1} \cdot F\right|+\left|\int_{D^{2}} G_{2} \cdot F\right|+\left|\int_{D^{2}} G_{3} \cdot F\right| \\
& \leq\left\|G_{1}\right\|_{W^{-1,(2, \infty)}}\|F\|_{W^{1,(2,1)}}+\left\|G_{2}\right\|_{W^{-1,2}}\|F\|_{W^{1,2}}+\left\|G_{3}\right\|_{L^{1}}\|F\|_{L^{\infty}} \\
& \lesssim\left(\left\|G_{1}\right\|_{W^{-1,(2, \infty)}}+\left\|G_{2}\right\|_{W^{-1,2}}+\left\|G_{3}\right\|_{L^{1}}\right)\|f\|_{L^{2,1}}
\end{aligned}
$$

Because $L^{2, \infty}$ is the dual space of $L^{2,1}$, the announced statement ensues.

\section{A.3. Miscellaneous identities}

We use in this section the same notation as that defined in the Introduction.

Lemma A.2. Let $\vec{\Phi}, \vec{n}$, and $\vec{H}$ be as in Theorem 2.1. We set

$$
Q:=\nabla \vec{H}-3 \pi_{\vec{n}}(\nabla \vec{H})+\star\left(\nabla^{\perp} \vec{n} \wedge \vec{H}\right) .
$$

Then the following identities hold:

$$
\nabla \vec{\Phi} \cdot Q=0 \quad \text { and } \quad \nabla \vec{\Phi} \wedge Q=-2 \nabla \vec{\Phi} \wedge \nabla \vec{H}
$$

Proof. We first note that ${ }^{11}$

$$
\nabla^{\perp} \vec{n}_{\alpha}=\left\langle\vec{e}_{1}, \nabla^{\perp} \vec{n}_{\alpha}\right\rangle \vec{e}_{1}+\left\langle\vec{e}_{2}, \nabla^{\perp} \vec{n}_{\alpha}\right\rangle \vec{e}_{2}+\left\langle\vec{n}_{\beta}, \nabla^{\perp} \vec{n}_{\alpha}\right\rangle \vec{n}_{\beta},
$$

so that

$$
\star\left(\vec{n} \wedge \nabla^{\perp} \vec{n}_{\alpha}\right)=\left\langle\vec{e}_{1}, \nabla^{\perp} \vec{n}_{\alpha}\right\rangle \vec{e}_{2}-\left\langle\vec{e}_{2}, \nabla^{\perp} \vec{n}_{\alpha}\right\rangle \vec{e}_{1} .
$$

Whence, since $\vec{n}_{\alpha} \wedge \vec{n}=0$,

$$
\star\left(\nabla^{\perp} \vec{n} \wedge \vec{n}_{\alpha}\right)=\left(\begin{array}{c}
h_{22}^{\alpha} \\
-h_{12}^{\alpha}
\end{array}\right) \partial_{x_{1}} \vec{\Phi}+\left(\begin{array}{c}
-h_{12}^{\alpha} \\
h_{11}^{\alpha}
\end{array}\right) \partial_{x_{2}} \vec{\Phi} .
$$

Accordingly, we find

$$
\nabla \vec{\Phi} \wedge \star\left(\nabla^{\perp} \vec{n} \wedge \vec{n}_{\alpha}\right)=\left(h_{12}^{\alpha}-h_{12}^{\alpha}\right) \partial_{x_{1}} \vec{\Phi} \wedge \partial_{x_{2}} \vec{\Phi}=0,
$$

and

$$
\nabla \vec{\Phi} \cdot \star\left(\nabla^{\perp} \vec{n} \wedge \vec{n}_{\alpha}\right)=\mathrm{e}^{2 \lambda}\left(h_{11}^{\alpha}+h_{22}^{\alpha}\right)=2 \mathrm{e}^{2 \lambda} H^{\alpha}
$$

\footnotetext{
${ }^{11}$ implicit summations over repeated indices are understood wherever appropriate.
} 
The last two identities yield

$$
\nabla \vec{\Phi} \wedge \star\left(\nabla^{\perp} \vec{n} \wedge \vec{H}\right)=0
$$

and

$$
\nabla \vec{\Phi} \cdot \star\left(\nabla^{\perp} \vec{n} \wedge \vec{H}\right)=2 \mathrm{e}^{2 \lambda}|\vec{H}|^{2} .
$$

For three indices $(a, b, c) \in\left\{x_{1}, x_{2}\right\}$, let

$$
F(a, b, c):=\partial_{a} \vec{\Phi} \wedge\left\langle\vec{e}_{b}, \partial_{c} \vec{H}\right\rangle \vec{e}_{b} .
$$

We have

$$
\begin{aligned}
F(a, b, c) & =\mathrm{e}^{\lambda}\left\langle\vec{e}_{b}, \partial_{c} \vec{H}\right\rangle \vec{e}_{a} \wedge \vec{e}_{b} \\
& =-\mathrm{e}^{\lambda} H^{\alpha}\left\langle\vec{n}_{\alpha}, \partial_{c} \vec{e}_{b}\right\rangle \vec{e}_{a} \wedge \vec{e}_{b} \quad \text { since } \vec{H}=H^{\alpha} \vec{n}_{\alpha} \\
& =-\mathrm{e}^{\lambda} H^{\alpha} h_{c b}^{\alpha} \vec{e}_{a} \wedge \vec{e}_{b} .
\end{aligned}
$$

Using this, we obtain

$$
\begin{aligned}
\text { (A.17) } \nabla \vec{\Phi} \wedge\left(\nabla \vec{H}-\pi_{\vec{n}}(\nabla \vec{H})\right) \equiv & \nabla \vec{\Phi} \wedge\left(\left\langle\vec{e}_{1}, \nabla \vec{H}\right\rangle \vec{e}_{1}+\left\langle\vec{e}_{2}, \nabla \vec{H}\right\rangle \vec{e}_{2}\right) \\
= & F\left(x_{1}, x_{1}, x_{1}\right)+F\left(x_{1}, x_{2}, x_{1}\right) \\
& +F\left(x_{2}, x_{1}, x_{2}\right)+F\left(x_{2}, x_{2}, x_{2}\right) \\
= & -\mathrm{e}^{\lambda} H^{\alpha} h_{21}^{\alpha} \vec{e}_{1} \wedge \vec{e}_{2}-\mathrm{e}^{\lambda} H^{\alpha} h_{12}^{\alpha} \vec{e}_{2} \wedge \vec{e}_{1} \\
= & 0 .
\end{aligned}
$$

Hence

(A.18) $\quad \nabla \vec{\Phi} \wedge\left(\nabla \vec{H}-3 \pi_{\vec{n}}(\nabla \vec{H})\right)=-2 \nabla \vec{\Phi} \wedge \nabla \vec{H}$

An evident yet nonetheless useful identity is

$$
\nabla \vec{\Phi} \cdot \pi_{\vec{n}}(\nabla \vec{H})=0
$$

thereby giving

$$
\nabla \vec{\Phi} \cdot\left(\nabla \vec{H}-3 \pi_{\vec{n}}(\nabla \vec{H})\right)=\nabla \vec{\Phi} \cdot \nabla \vec{H}
$$

Bringing altogether (A.15) and (A.18) yields the second part of (A.13). Deriving the first part of (A.13) requires a bit more work. Combining (A.16) 
and (A.19) shows that

$$
\nabla \vec{\Phi} \cdot Q=\nabla \vec{\Phi} \cdot \nabla \vec{H}+2 \mathrm{e}^{2 \lambda}|\vec{H}|^{2}
$$

For three indices $(a, b, c) \in\left\{x_{1}, x_{2}\right\}$, let

$$
G(a, b, c):=\partial_{a} \vec{\Phi} \cdot\left\langle\vec{e}_{b}, \partial_{c} \vec{H}\right\rangle \vec{e}_{b}
$$

Just as above, we verify easily that

$$
G(a, b, c)=-\mathrm{e}^{2 \lambda} H^{\alpha} h_{c b}^{\alpha} \delta_{a b} .
$$

Consequently, there holds

$$
\begin{aligned}
\nabla \vec{\Phi} \cdot \nabla \vec{H} & =G\left(x_{1}, x_{1}, x_{1}\right)+G\left(x_{1}, x_{2}, x_{1}\right)+G\left(x_{2}, x_{1}, x_{2}\right)+G\left(x_{2}, x_{2}, x_{2}\right) \\
& =-\mathrm{e}^{2 \lambda} H^{\alpha} h_{11}^{\alpha}-\mathrm{e}^{2 \lambda} H^{\alpha} h_{22}^{\alpha} \\
& =-2 \mathrm{e}^{2 \lambda}|\vec{H}|^{2} .
\end{aligned}
$$

Putting this into (A.20) finally gives the first sought after identity in (A.13).

Lemma A.3. Using the notation introduced in Section 3.2.2, the CodazziMainardi identity may be recast in the form

$$
\mathrm{e}^{-2 \lambda} \partial_{\bar{z}}\left(e^{2 \lambda} \overline{\vec{H}_{0}} \cdot \vec{H}\right)=\vec{H} \cdot \partial_{z} \vec{H}+\overline{\vec{H}_{0}} \cdot \partial_{\bar{z}} \vec{H}
$$

Proof. Consider the 1-form

$$
\eta_{\alpha}:=\left\langle\vec{e}_{\bar{z}}, d \vec{n}_{\alpha}\right\rangle
$$

On one hand, from (3.43), there holds

$$
\eta_{\alpha}=\left(\vec{e}_{\bar{z}} \cdot \partial_{z} \vec{n}_{\alpha}\right) d z+\left(\vec{e}_{\bar{z}} \cdot \partial_{\bar{z}} \vec{n}_{\alpha}\right) d \bar{z}=-\frac{\mathrm{e}^{\lambda}}{2}\left(H^{\alpha} d z+H_{0}^{\alpha} d \bar{z}\right)
$$

so that

$$
\star d \eta_{\alpha}=-\frac{\mathrm{e}^{\lambda}}{2}\left(H_{0}^{\alpha} \partial_{z} \lambda-H^{\alpha} \partial_{\bar{z}} \lambda+\partial_{z} H_{0}^{\alpha}-\partial_{\bar{z}} H^{\alpha}\right)
$$


On the other hand, with (3.42) and (3.43), we find

$$
\begin{aligned}
\star d \eta_{\alpha} & =\star d\left\langle\vec{e}_{\bar{z}}, d \vec{n}_{\alpha}\right\rangle=\partial_{z} \vec{e}_{\bar{z}} \cdot \partial_{\bar{z}} \vec{n}_{\alpha}-\partial_{\bar{z}} \vec{e}_{\bar{z}} \cdot \partial_{z} \vec{n}_{\alpha} \\
& =\frac{\mathrm{e}^{\lambda}}{2}\left(H_{0}^{\alpha} \partial_{z} \lambda+H^{\alpha} \partial_{\bar{z}} \lambda+\vec{H} \cdot \partial_{\bar{z}} \vec{n}_{\alpha}-\vec{H}_{0} \cdot \partial_{z} \vec{n}_{\alpha}\right) .
\end{aligned}
$$

Comparing the latter to (A.22) yields

$$
2 H_{0}^{\alpha} \partial_{z} \lambda=\partial_{\bar{z}} H^{\alpha}-\partial_{z} H_{0}^{\alpha}-\vec{H} \cdot \partial_{\bar{z}} \vec{n}_{\alpha}+\vec{H}_{0} \cdot \partial_{z} \vec{n}_{\alpha}
$$

By antisymmetry, there holds

$$
H^{\alpha} \vec{H} \cdot \partial_{\bar{z}} \vec{n}_{\alpha}=H^{\alpha} H^{\beta} \vec{n}_{\beta} \cdot \partial_{\bar{z}} \vec{n}_{\alpha}=0
$$

and whence

$$
H^{\alpha}\left(\partial_{\bar{z}} H^{\alpha}-\vec{H} \cdot \partial_{\bar{z}} \vec{n}_{\alpha}\right)=H^{\alpha}\left(\partial_{\bar{z}} H^{\alpha}+\vec{H} \cdot \partial_{\bar{z}} \vec{n}_{\alpha}\right) \equiv \vec{H} \cdot \partial_{\bar{z}} \vec{H}
$$

Similarly, we find

$$
\begin{aligned}
H^{\alpha}\left(\partial_{z} H_{0}^{\alpha}-\vec{H}_{0} \cdot \partial_{z} \vec{n}_{\alpha}\right) & =\partial_{z}\left(\vec{H}_{0} \cdot \vec{H}\right)-H_{0}^{\alpha} \partial_{z} H^{\alpha}-H^{\alpha} \vec{H}_{0} \cdot \partial_{z} \vec{n}_{\alpha} \\
& =\partial_{z}\left(\vec{H}_{0} \cdot \vec{H}\right)-\vec{H}_{0} \cdot \partial_{z} \vec{H}
\end{aligned}
$$

Multiplying (A.23) throughout by $H^{\alpha}$, summing over $\alpha$, and using (A.24) and (A.25) gives

$$
2 \vec{H} \cdot \vec{H}_{0} \partial_{z} \lambda=-\partial_{z}\left(\vec{H} \cdot \vec{H}_{0}\right)+\vec{H} \cdot \partial_{\bar{z}} \vec{H}+\vec{H}_{0} \cdot \partial_{z} \vec{H}
$$

from which the (complex conjugate of the) desired (A.21) ensues.

Lemma A.4. Using the notation of Section 3.2, there holds

$$
-2 \Delta \vec{\Phi}=\nabla \vec{R} \bullet \nabla^{\perp} \vec{\Phi}+\nabla S \cdot \nabla^{\perp} \vec{\Phi}
$$

Proof. Note that for any 1 -vector $\vec{a}$, we have

$$
\left(\vec{a} \wedge \vec{e}_{j}\right) \bullet \vec{e}_{i}=\left(\vec{e}_{i}\llcorner\vec{a}) \wedge \vec{e}_{j}+\vec{a} \wedge\left(\vec{e}_{i}\left\llcorner\vec{e}_{j}\right)=\left(\vec{e}_{i} \cdot \vec{a}\right) \vec{e}_{j}+\delta_{i j} \vec{a}\right.\right.
$$


From this, and $\vec{e}_{i}:=\mathrm{e}^{-\lambda} \partial_{x_{i}} \vec{\Phi}$, it follows that whenever

$$
\vec{V}:=V^{i} \vec{e}_{i}+V^{\alpha} \vec{n}_{\alpha}
$$

then

$$
\left\{\begin{array}{l}
\left(\vec{V} \wedge \nabla^{\perp} \vec{\Phi}\right) \bullet \nabla^{\perp} \vec{\Phi}=\mathrm{e}^{2 \lambda}\left(3 V^{i} \vec{e}_{i}+2 V^{\alpha} \vec{n}_{\alpha}\right) \\
(\vec{V} \wedge \nabla \vec{\Phi}) \bullet \nabla^{\perp} \vec{\Phi}=\mathrm{e}^{2 \lambda}\left(V^{2} \vec{e}_{1}-V^{1} \vec{e}_{2}\right) \equiv(\vec{V} \cdot \nabla \vec{\Phi}) \cdot \nabla^{\perp} \vec{\Phi}
\end{array}\right.
$$

In particular, since $\vec{H}=H^{\alpha} \vec{n}_{\alpha}$, we find that

$$
\begin{aligned}
\nabla \vec{R} \bullet \nabla^{\perp} \vec{\Phi} & \equiv-\left(\vec{L} \wedge \nabla \vec{\Phi}+2 \vec{H} \wedge \nabla^{\perp} \vec{\Phi}\right) \bullet \nabla^{\perp} \vec{\Phi} \\
& =-(\vec{L} \cdot \nabla \vec{\Phi}) \cdot \nabla^{\perp} \vec{\Phi}-4 \mathrm{e}^{2 \lambda} \vec{H} \\
& =-\nabla S \cdot \nabla^{\perp} \vec{\Phi}-4 \mathrm{e}^{2 \lambda} \vec{H}
\end{aligned}
$$

Hence,

$$
\nabla \vec{R} \bullet \nabla^{\perp} \vec{\Phi}+\nabla S \cdot \nabla^{\perp} \vec{\Phi}=-4 \mathrm{e}^{2 \lambda} \vec{H}
$$

Finally, there remains to recall that

$$
\Delta \vec{\Phi}=2 \mathrm{e}^{2 \lambda} \vec{H}
$$

to reach the desired identity (A.26).

\section{References}

[1] R. Adams, Sobolev Spaces, Academic Press, New York, 2003.

[2] R. Adams, A note on Riesz potentials, Duke Math. J. 42(4) (1975), 765-778.

[3] J. Bergh and J. Löfström, Interpolation spaces: an introduction, Springer, Berlin, 1976.

[4] Y. Bernard and T. Rivière, Asymptotic analysis of branched Willmore surfaces, to appear. arXiv 1106:4642, June 2011.

[5] F. Bethuel, Un résultat de régularité pour les solutions de l'équation de surface à courbure moyenne prescrite, C. R. Acad. Sci. Paris Série I Math. 314(13) (1992), 1003-1007. 
[6] C. Bohle, G.P. Peters and U. Pinkall, Constrained Willmore surfaces, Calc. Var. Partial Differential Equations 32 (2008), 263-277.

[7] H. Brezis, and S. Wainger A note on limiting cases of Sobolev embeddings and convolutions inequalities, Comm. Partial Differential Equations 5 (1980), 773-789.

[8] F. Burstall, F. Pedit and U. Pinkall, Schwarzian derivatives and flows of surfaces, Contemp. Math. 308 (2002), 39-61.

[9] B.-Y. Chen, Some conformal invariants of submanifolds and their applications, Boll. Un. Mat. Ital. (4) 10 (1974), 380-385.

[10] R. Coifman, P.-L. Lions, Y. Meyer and S. Semmes, Compensated compactness and Hardy spaces, J. Math. Pures Appl. (9) 72(3) (1993), 247-286.

[11] G. Friesecke, R.D. James, and S. Müller, A theorem on geometric rigidity and the derivation of nonlinear plate theory from three-dimensional elasticity, Comm. Pure Appl. Math. 55(11) (2002), 1461-1506.

[12] Y. Ge, A remark on generalized harmonic maps into the sphere, Nonlinear Anal., T.M.A. 36 (1999), 495-506.

[13] S.W. Hawking, Gravitational radiation in an expanding universe, J. Math. Phys. 9 (1968), 598-604.

[14] F. Hélein, Harmonic maps, conservation laws, and moving frames, Cambridge Tracts Math., 150, Cambridge University Press, Cambridge, 2002.

[15] W. Helfrich, Elastic properties of lipid bilayers: theory and possible experiments, Z. Naturforsch. C28 (1973), 693-703.

[16] G. Huisken, T. Ilmanen, The Riemannian Penrose inequality, Int. Math. Res. Notes 20 (1997), 1045-1058.

[17] R.A. Hunt, On $L(p, q)$ spaces, L'enseignement Mathématique XII (1966), 249-276.

[18] J. Jost, Compact Riemann surfaces, Universitext, Springer 2006.

[19] S. Müller, V. Šverák, On surfaces of finite total curvature, J. Differential Geom. 42(2) (1995), 229-258.

[20] U. Pinkall, I. Sterling, Willmore surfaces, Math. Intell. 9(2) (1987), 38-43. 
[21] A.M. Polyakov, Fine structure of strings, Nucl. Phys. B268 (1986), 1183.

[22] J. Richter, Conformal maps of a Riemann surface into the space of quaternions, Thesis, TU-Berlin, 1997.

[23] T. Rivière, Conservation laws for conformally invariant variational problems, Invent. Math. 168(1) (2006), 1-22.

[24] T. Rivière, Analysis aspects of the Willmore functional, Invent. Math. 174(1) (2008), 1-45.

[25] T. Rivière, Variational principles for immersed surfaces with $L^{2}$ bounded second fundamental form, arXiv:1007.2997, July 2010.

[26] L. Simon, Existence of surfaces minimizing the Willmore functional, Comm. Anal. Geom. 1(2) (1993), 281-326.

[27] L. Tartar, An introduction to Sobolev spaces and interpolation spaces, Lectures notes of the Unione Matematica Italiana, 2007.

[28] L. Tartar, Remarks on oscillations and Stokes' equation. Macroscopic modelling of turbulent flows (Nice, 1984), 24-31, Lecture Notes in Phys., 230, Springer, Berlin, 1985.

[29] L. Tartar, Imbedding theorems of Sobolev spaces into Lorentz spaces, Boll. Unione Mat. Ital. Sez. B Artic. Ric. Mat. (8) 1(3) (1998), 479500 .

[30] T. Toro, Surfaces with generalized second fundamental form in $L^{2}$ are Lipschitz manifolds J. Differential Geom. 39 (1994), 65-101.

[31] T. Toro, Geometric conditions and existence of bilipschitz parametrisations, Duke Math. J. 77(1) (1995), 193-227.

[32] J. Weiner, On a problem of Chen, Willmore, et al., Indiana U. Math. J. $27(1)$ (1978), 19-35.

[33] H.C. Wente, An existence theorem for surfaces of constant mean curvature, J. Math. Anal. Appl. 26 (1969), 318-344.

[34] J.H. White, A global invariant of conformal mappings in space, Proc. Amer. Math. Soc. 38 (1973), 162-164.

[35] T.J. Willmore, Note on embedded surfaces, Ann. Stiint. Univ. "Al. I. Cuza" Iasi. Sect. I a Mat. (N.S.) 11B (1965), 493-496. 
[36] T. J. Willmore, Riemannian geometry, Oxford University Press, Oxford, 1997.

Mathematisches Institut

Albert-Ludwigs-UniversitäT

79004 FREIBURG

GERMANY

E-mail address: yann.bernard@math.uni-freiburg.de

Department of Mathematics

ETH ZENTRUM

8093 ZÜRICH

SWITZERLAND

E-mail address: tristan.riviere@fim.math.ethz.ch

Upon the completion of this work, the first author held a position at the ETH, Zürich.

Received May 27, 2009 
